NISSUNA UMANA INVESTIGAZIONE SI PUO DIMANDARE VERA SCIENZIA S'ESSA NON PASSA PER LE MATEMATICHE DIMOSTRAZIONI LEONARDO DA VINCI

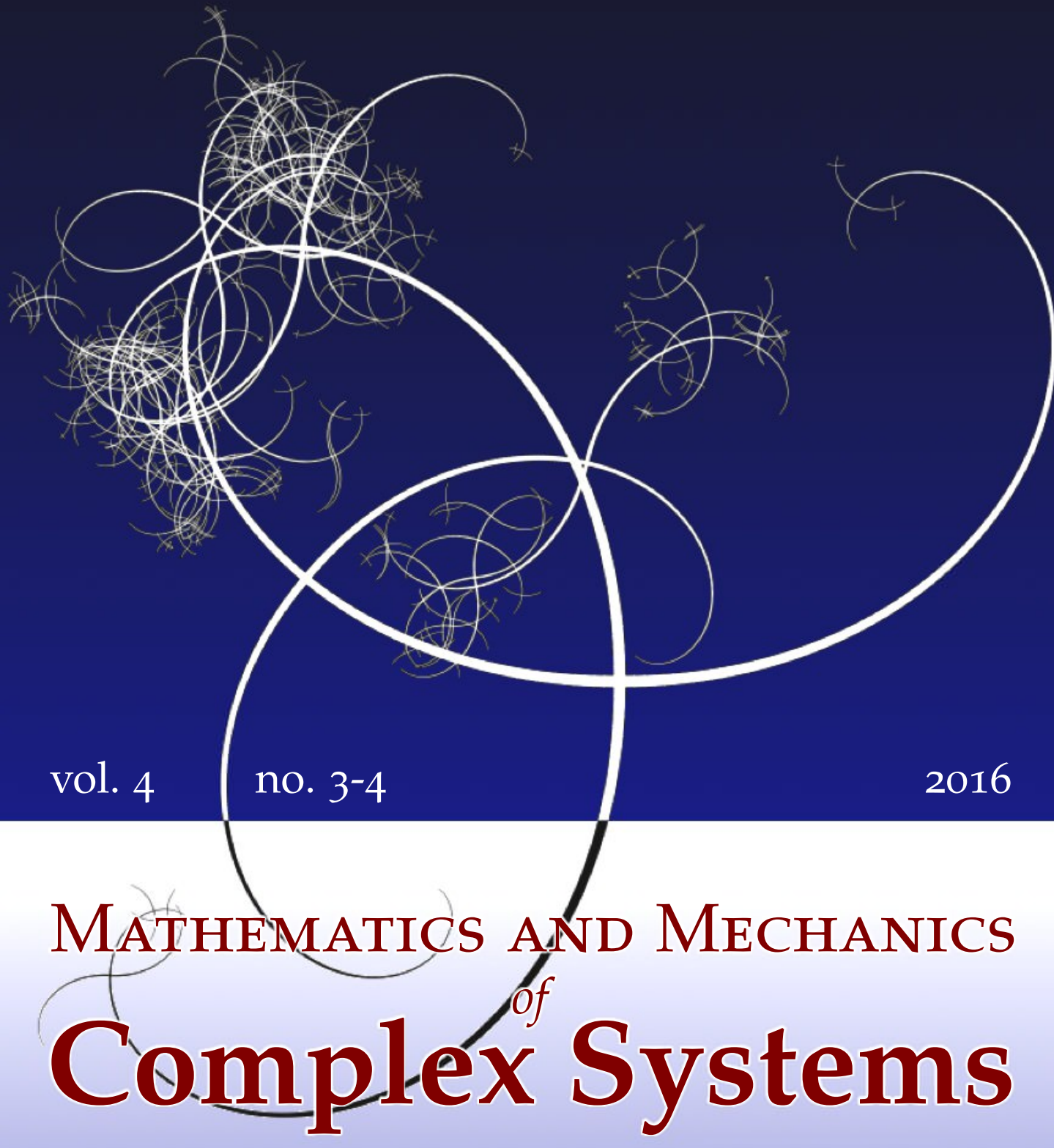

ViNCENZO CAPASSO AND FRANCO FLANDOLI ON STOCHASTIC DISTRIBUTIONS AND CURRENTS 


\title{
ON STOCHASTIC DISTRIBUTIONS AND CURRENTS
}

\author{
VinCENZO CAPASSO AND FRANCO FLANDOLI \\ Dedicated to Lucio Russo, on the occasion of his 70th birthday
}

\begin{abstract}
In many applications, it is of great importance to handle random closed sets of different (even though integer) Hausdorff dimensions, including local information about initial conditions and growth parameters. Following a standard approach in geometric measure theory, such sets may be described in terms of suitable measures. For a random closed set of lower dimension with respect to the environment space, the relevant measures induced by its realizations are singular with respect to the Lebesgue measure, and so their usual Radon-Nikodym derivatives are zero almost everywhere. In this paper, how to cope with these difficulties has been suggested by introducing random generalized densities (distributions) á la Dirac-Schwarz, for both the deterministic case and the stochastic case. For the last one, mean generalized densities are analyzed, and they have been related to densities of the expected values of the relevant measures. Actually, distributions are a subclass of the larger class of currents; in the usual Euclidean space of dimension $d$, currents of any order $k \in\{0,1, \ldots, d\}$ or $k$ currents may be introduced. In this paper, the cases of 0-currents (distributions), 1-currents, and their stochastic counterparts are analyzed. Of particular interest in applications is the case in which a 1-current is associated with a path (curve). The existence of mean values has been discussed for currents too. In the case of 1-currents associated with random paths, two cases are of interest: when the path is differentiable, and also when it is the path of a Brownian motion or (more generally) of a diffusion. Differences between the two cases have been discussed, and nontrivial problems are mentioned which arise in the case of diffusions. Two significant applications to real problems have been presented too: tumor driven angiogenesis, and turbulence.
\end{abstract}

\section{Introduction: preliminaries and notation}

Many real phenomena may be modeled as random closed sets in $\mathbb{R}^{d}$, and in several situations as evolving random closed sets. Application areas include crystallization processes: Figures 1 and 2 (see [Capasso 2003; Capasso and Micheletti 2006], and

\section{Communicated by Raffaele Esposito.}

MSC2010: 28A75, 47B80, 60D05, 60H25, 46FXX, 52A22, 53C65.

Keywords: stochastic geometry, random distributions, random currents, mean geometric densities. 


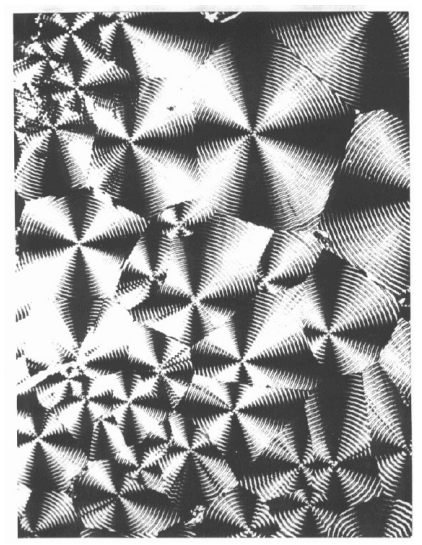

Figure 1. The final tessellation in a real experiment of a crystallization process of a polymer [MONTELL-Italy].

references therein; see also [Ubukata 2003] for the crystallization processes on sea shells, and [Callister Jr. 2007, p. 92; Hochrainer et al. 2007; Kassner et al. 2000] for dislocations: Figure 3); tumor growth [Anderson 2003]; angiogenesis: Figure 6 [Carmeliet and Jain 2000]; patterns in biology: Figure 4; the spread of a pollutant in an environment; etc.

All quoted processes may be described by time dependent random closed sets at different Hausdorff dimensions (for instance, crystallization processes are modeled in general by full dimensional growing sets and lower dimensional interfaces, while angiogenesis by systems of random curves). In many cases, because of the coupling with suitable underlying fields (such as temperature, nutrients, etc.), these kinds of phenomena may be modeled as space-time structured stochastic processes, whose geometric structure is of great relevance, as discussed in [Capasso et al. 2013].

A rigorous definition of the relevant geometric quantities in a stochastic setting of the above systems (fibers for angiogenesis, dislocations for crystalline materials, etc.) is very important for statistical applications (see, e.g., [Ambrosio et al. 2009; Camerlenghi et al. 2014]), and in mean field approximations (see, e.g., [Bonilla et al. 2017; Hochrainer et al. 2007; Bessaih et al. 2017]).

A presentation of an angiogenesis model will be offered later in Section 4.1.

For definitions and basic properties of Hausdorff measure and Hausdorff dimension see, e.g., [Ambrosio et al. 2000; Falconer 1986; Federer 1996; Morgan 1998].

We remind here the concepts and results of current literature which are relevant for our analysis. Actually, the subject of stochastic geometry, considered here in the direction of geometric measure theory, does have a nontrivial intersection with the literature on convex geometry for which the reader may refer to [Baddeley et al. 2007] and references therein. 


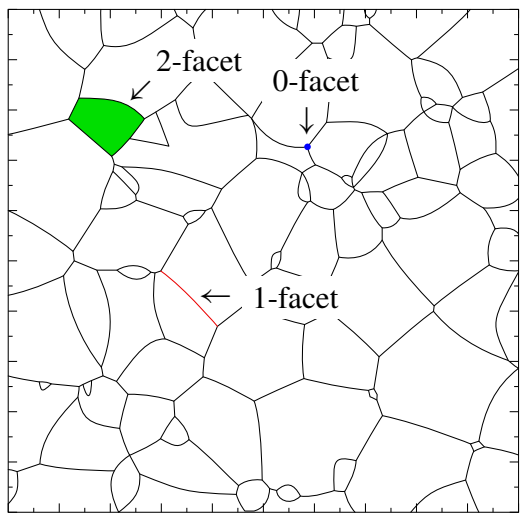

Figure 2. The final tessellation in a simulated experiment of a crystallization process of a polymer. This picture, together with the real one, shows the relevance of components at all integer Hausdorff dimensions for describing the final morphology [Burger et al. 2002].

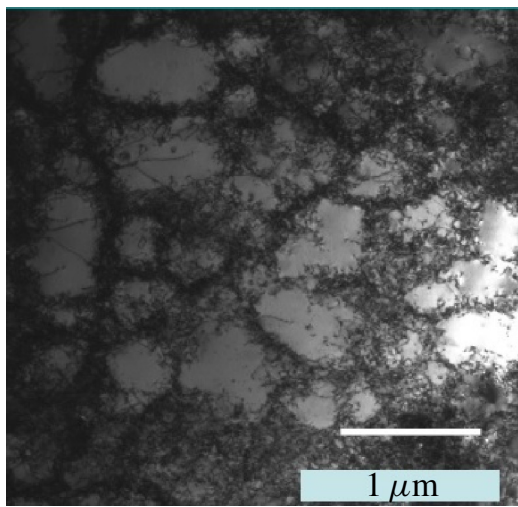

Figure 3. Dislocations in copper crystals [M. Kassner; private collection].

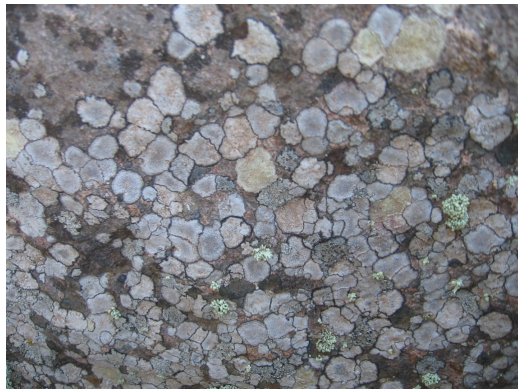

Figure 4. Pattern formation in a lichen colony [V. Capasso; private collection]. 


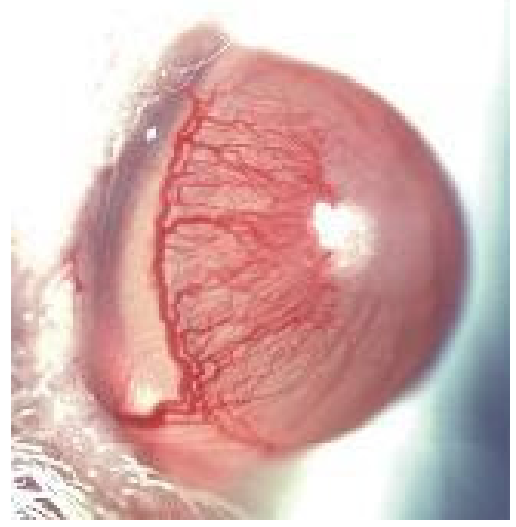

Figure 5. Angiogenesis on a rat cornea [Dejana, personal collection].

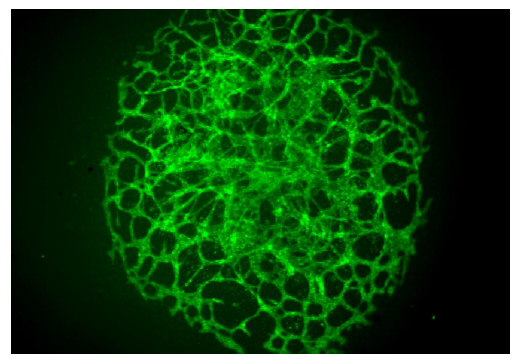

Figure 6. Vascularization of an allantoid. [Dejana, personal collection]. An important example of a fiber process of Hausdorff dimension 1 in a 3D space.

Let us consider the space $\mathbb{R}^{d}$ for $d>1$, and denote by $v^{d}$ the usual $d$-dimensional Lebesgue measure, and by $\mathcal{B}_{\mathbb{R}^{d}}$ the Borel $\sigma$-algebra of $\mathbb{R}^{d}$.

We know that every positive Radon measure $\mu$ on $\mathbb{R}^{d}$ can be represented as

$$
\mu=\mu_{\ll}+\mu_{\perp},
$$

where $\mu_{\ll}$ and $\mu_{\perp}$ are the absolutely continuous part with respect to $v^{d}$ and the singular part of $\mu$, respectively. We shall denote by $B_{r}(x)$ the $d$-dimensional closed ball centered in $x$ with radius $r$.

Let us denote by $\mathcal{H}^{s}$ the $s$-dimensional Hausdorff measure.

We will consider a class of subsets of $\mathbb{R}^{d}$ with integer Hausdorff dimension.

Definition 1. Given an integer $n \in[0, d]$, we say that a closed subset $A$ of $\mathbb{R}^{d}$ is $n$-regular if it satisfies the following conditions:

(i) $\mathcal{H}^{n}\left(A \cap B_{R}(0)\right)<\infty$ for any $R>0$; 
(ii) $\lim _{r \rightarrow 0} \frac{\mathcal{H}^{n}\left(A \cap B_{r}(x)\right)}{b_{n} r^{n}}=1$ for $\mathcal{H}^{n}$-a.e. $x \in A$.

We recall that when $n$ is integer, then $b(n)=b_{n}$, which is the volume of the unit ball in $\mathbb{R}^{n}$.

Note that condition (ii) is related to a characterization of the $\mathcal{H}^{n}$-rectifiability of the set $A$ [Falconer 1986, p.256, 267; Ambrosio et al. 2000, p.83].

Remark 2. We may observe that if $\Theta$ is an $n$-regular closed set in $\mathbb{R}^{d}$, we have

$$
\lim _{r \rightarrow 0} \frac{\mathcal{H}^{n}\left(\Theta \cap B_{r}(x)\right)}{b_{n} r^{n}}= \begin{cases}1 & \mathcal{H}^{n} \text {-a.e. } x \in \Theta \\ 0 & \text { for } x \notin \Theta .\end{cases}
$$

In fact, since $\Theta^{C}$ is open, for all $x \notin \Theta$ there exists $r_{0}>0$ such that for all $r \leq r_{0}$, we have $B_{r}(x) \subset \Theta^{C}$, that is $\mathcal{H}^{n}\left(\Theta \cap B_{r}(x)\right)=0$ for all $r \leq r_{0}$; thus the limit equals 0 for all $x \in \Theta^{C}$.

For a general set $A$, problems about " $\mathcal{H}^{n}$-a.e." and "for all" may arise when we consider a point $x \in \partial A$ where the boundary is not a regular manifold. For example, if $A$ is a closed square in $\mathbb{R}^{2}$, for all points $x$ on the edges,

$$
\lim _{r \rightarrow 0} \frac{\mathcal{H}^{2}\left(A \cap B_{r}(x)\right)}{b_{2} r^{2}}=\frac{1}{2},
$$

while for each of the four vertices the limit equals $\frac{1}{4}$.

Observe that in both cases the set of such points has $\mathcal{H}^{2}$-measure zero.

From now on we shall consider $n$-regular closed sets $\Theta$ in $\mathbb{R}^{d}$, with $0 \leq n \leq d$. As a consequence, for $n<d$ (by assuming $0 \cdot \infty=0$ ) by (1-1) we also have

$$
\lim _{r \rightarrow 0} \frac{\mathcal{H}^{n}\left(\Theta \cap B_{r}(x)\right)}{b_{d} r^{d}}=\lim _{r \rightarrow 0} \frac{\mathcal{H}^{n}\left(\Theta \cap B_{r}(x)\right)}{b_{n} r^{n}} \frac{b_{n} r^{n}}{b_{d} r^{d}}= \begin{cases}\infty & \mathcal{H}^{n} \text {-a.e. } x \in \Theta, \\ 0 & \text { for } x \notin \Theta .\end{cases}
$$

Note that in the particular case $n=0$, with $\Theta=X_{0}$ point in $\mathbb{R}^{d}\left(X_{0}\right.$ is indeed a 0 -regular closed set),

$$
\lim _{r \rightarrow 0} \frac{\mathcal{H}^{0}\left(X_{0} \cap B_{r}(x)\right)}{b_{d} r^{d}}= \begin{cases}\infty & \text { if } x=X_{0}, \\ 0 & \text { if } x \neq X_{0} .\end{cases}
$$

Note that if $\Theta$ is an $n$-regular closed set in $\mathbb{R}^{d}$ with $n<d$, then the Radon measure

$$
\mu_{\Theta}(\cdot):=\mathcal{H}^{n}(\Theta \cap \cdot)
$$

is a singular measure with respect to $v^{d}$.

It is then clear that the quantity

$$
\delta_{\Theta}(x):=\lim _{r \rightarrow 0} \frac{\mathcal{H}^{n}\left(\Theta \cap B_{r}(x)\right)}{b_{d} r^{d}}, \quad x \in \mathbb{R}^{d},
$$


associated with $\Theta$ cannot be considered as a classical function. But, analogously to the Dirac delta function $\delta_{X_{0}}(x)$ associated with a point $X_{0} \in \mathbb{R}^{d}$, we may refer to it as a generalized density (or the generalized Radon-Nikodym derivative of the measure $\mu_{\Theta}$ with respect to $v_{d}$ ), or better as a distribution á la Schwarz (see Section 2).

We may notice that in the case $\Theta=X_{0}$, the generalized density $\delta_{X_{0}}(x)$ coincides with the well-known Dirac delta function at a point $X_{0}$ that is the (generalized) density of the singular Dirac measure $\varepsilon_{X_{0}}$ [Kolmogorov and Fomin 1970].

The usefulness of introducing these generalized functions associated with sets of any dimension $n \in\{0, \ldots, d\}$, in particular in the stochastic case, has been discussed in various papers [Matheron 1965; Ambrosio et al. 2009; Burger et al. 2002; Capasso and Villa 2006; 2007; Vladimirov 1979].

The previous ideas extend to another framework, the one of currents. First, let us restrict ourselves to the so-called 1-currents, which heuristically are distributional generalizations of the concept of vector fields. In the smooth case, a 1-current in $\mathbb{R}^{d}$ is simply a smooth vector field $\xi: \mathbb{R}^{d} \rightarrow \mathbb{R}^{d}$. A smooth vector field $\xi(x)$ acts on test functions (above we have introduced objects using the language of measures, but here it is more natural to use directly the language of test functions) as

$$
(\xi, \theta)=\int_{\mathbb{R}^{d}}\langle\xi(x), \theta(x)\rangle \mathrm{d} x,
$$

for all smooth test vector fields $\theta: \mathbb{R}^{d} \rightarrow \mathbb{R}^{d}$. But, similarly to the case of measures concentrated on lower-dimensional subsets $\Theta$ of $\mathbb{R}^{d}$, we may consider vector fields concentrated on lower-dimensional subsets. Let us restrict further our discussion of 1-currents to the case of currents associated with curves. Given a smooth curve $\gamma:[a, b] \rightarrow \mathbb{R}^{d}$, the concept of current associates to it a distribution vector field concentrated along the curve, and having the direction tangent to the curve. The action on smooth test vector fields $\theta: \mathbb{R}^{d} \rightarrow \mathbb{R}^{d}$ is

$$
(\xi, \theta)=\int_{a}^{b}\left\langle\theta(\gamma(t)), \gamma^{\prime}(t)\right\rangle \mathrm{d} t,
$$

or, in the more intuitive language that we shall explain in the sequel,

$$
\xi(x)=\int_{a}^{b} \delta_{\gamma(t)}(x) \gamma^{\prime}(t) \mathrm{d} t,
$$

which emphasizes the fact that the vector field is localized on the curve, with the direction of the tangent.

In Section 2 we present densities of measures as linear functionals for both the deterministic and the stochastic case; special emphasis is given to random functionals associated with random closed sets and their mean values. Section 3 is devoted 
to 1-currents with their stochastic counterparts. Finally Section 4 presents examples of significant applications of real interest, such as tumor driven angiogenesis and turbulence.

\section{Densities as linear functionals}

2.1. The deterministic case. We know that the Dirac delta $\delta_{X_{0}}$ at a point $X_{0} \in \mathbb{R}^{d}$ can be defined as a linear functional associated with a finite Borel measure, the well-known Dirac measure $\varepsilon_{X_{0}}$, concentrated at $X_{0}$; as such it is the (generalized) density of $\varepsilon_{X_{0}}$. In fact, we recall that, according to the Riesz representation theorem (see, e.g., [Folland 1999, p. 212]), Radon measures in $\mathbb{R}^{d}$ (i.e., nonnegative and $\sigma$ additive set functions defined on the Borel $\sigma$-algebra $\mathcal{B}_{\mathbb{R}^{d}}$ that are finite on bounded sets) can be canonically identified with linear and order preserving functionals on $C_{c}\left(\mathbb{R}^{d}, \mathbb{R}\right)$, the space of continuous functions with compact support in $\mathbb{R}^{d}$. The identification is provided by the integral operator, i.e.,

$$
(\mu, f)=\int_{\mathbb{R}^{d}} f \mathrm{~d} \mu \quad \forall f \in C_{c}\left(\mathbb{R}^{d}, \mathbb{R}\right) .
$$

If $\mu \ll v^{d}$, it admits (as a Radon-Nikodym density) a classical function $\delta_{\mu}$ defined almost everywhere in $\mathbb{R}^{d}$, so that

$$
(\mu, f)=\int_{\mathbb{R}^{d}} f(x) \delta_{\mu}(x) \mathrm{d} x \quad \forall f \in C_{c}\left(\mathbb{R}^{d}, \mathbb{R}\right),
$$

in the usual sense of a Lebesgue integral.

If $\mu \perp v^{d}$, we may speak of a density $\delta_{\mu}$ only in the sense of distributions (formally, it is almost everywhere trivial, but it is $\infty$ on a set of $v^{d}$-measure zero). In this case, the symbol

$$
\int_{\mathbb{R}^{d}} f(x) \delta_{\mu}(x) \mathrm{d} x:=(\mu, f)
$$

can still be adopted, provided the integral on the left-hand side is understood in a generalized sense, and not as a Lebesgue integral.

In either case, from now on, we may denote by $\left(\delta_{\mu}, f\right)$ the quantity $(\mu, f)$. Accordingly, we say that a sequence of measures $\mu_{n}$ weakly* converges to a Radon measure $\mu$ if $\left(\delta_{\mu_{n}}, f\right)$ converges to $\left(\delta_{\mu}, f\right)$ for any $f \in C_{c}\left(\mathbb{R}^{d}, \mathbb{R}\right)$. A classical criterion (see, for instance, [Evans and Gariepy 1992, p. 54; Ambrosio et al. 2000]) states that $\mu_{n}$ weakly* converge to $\mu$ if and only if $\mu_{n}(A) \rightarrow \mu(A)$ for any bounded open set $A$ with $\mu(\partial A)=0$.

By the common integral representation for generalized functions,

$$
\int_{A} \delta_{X_{0}}(x) \mathrm{d} x:=\varepsilon_{X_{0}}(A)=\mathcal{H}^{0}\left(X_{0} \cap A\right),
$$


we have

$$
\left(\delta_{X_{0}}, f\right)=\int_{\mathbb{R}^{d}} \delta_{X_{0}}(x) f(x) \mathrm{d} x=f\left(X_{0}\right), \quad f \in C_{c}\left(\mathbb{R}^{d}, \mathbb{R}\right) .
$$

Now we are ready to introduce the delta function of an $n$-regular set $\Theta$ as the linear functional (the generalized function) $\delta_{\Theta}$ in a similar way.

Consider the measure defined on the Borel $\sigma$-algebra of $\mathbb{R}^{d}$, as follows:

$$
\mu_{\Theta}(A):=\mathcal{H}^{n}(\Theta \cap A), \quad A \in \mathcal{B}_{\mathbb{R}^{d}} .
$$

The linear functional associated with it is

$$
\left(\mu_{\Theta}, f\right):=\int_{\mathbb{R}^{d}} f(x) \mu_{\Theta} \mathrm{d} x
$$

for $f \in C_{c}\left(\mathbb{R}^{d}, \mathbb{R}\right)$.

In accordance with what we have said in the Introduction, the following holds.

Proposition 3. If $n$ is an integer strictly less than $d p$ the measure $\mu_{\Theta}$ is a singular measure with respect to the usual Lebesgue measure on $\mathbb{R}^{d}$.

In accordance with the usual representation of distributions in the theory of generalized functions, we formally write

$$
\int_{\mathbb{R}^{d}} f(x) \mu_{\Theta} \mathrm{d} x=\int_{\mathbb{R}^{d}} f(x) \delta_{\Theta}(x) \mathrm{d} x=:\left(\delta_{\Theta}, f\right) .
$$

Remark 4. We may notice that the classical Dirac delta $\delta_{X_{0}}(x)$ associated to a point $X_{0}$ is now a particular case corresponding to $n=0$. If $\Theta$ is a piecewise smooth surface $S$ in $\mathbb{R}^{d}$ (and so 2-regular), then by the definition in (2-2), it follows that for any test function $f$,

$$
\left(\delta_{S}, f\right)=\int_{S} f(x) \mathrm{d} S,
$$

which is the definition of $\delta_{S}$ in [Vladimirov 1979, p. 33].

In terms of the above arguments, we may state that $\delta_{\Theta}(x)$ is the (generalized) density of the measure $\mu_{\Theta}$, defined by (2-1), with respect to the usual Lebesgue measure $v^{d}$ on $\mathbb{R}^{d}$. Note that if $n=d$, then $\mu_{\Theta}$ is absolutely continuous with respect to $v^{d}$, so that $\delta_{\Theta}$ is its classical Radon-Nikodym derivative [Kolmogorov 1956].

\subsubsection{Paths: 1-dimensional sets.}

Definition 5. A path (or curve or line) in $\mathbb{R}^{d}$ is a continuous mapping $\gamma:[a, b] \subset$ $\mathbb{R} \rightarrow \mathbb{R}^{d}$. The point $\gamma(a)$ is called the initial point, and the point $\gamma(b)$ is called the final point. The image of the path $\gamma([a, b])$ is called the arc or the support of $\gamma$. A path $\gamma$ is closed if its end points coincide; it is simple if it has no multiple points (apart from possibly the end points for a closed path). 
If we denote by $e_{j}: j=1, \ldots, d$ the canonical basis of $\mathbb{R}^{d}$, we may introduce the components of the vector function $\gamma$ as

$$
\gamma_{j}=\left\langle e_{j}, \gamma\right\rangle:[a, b] \rightarrow \mathbb{R} .
$$

For $t \in(a, b)$, we put

$$
\gamma^{\prime}(t):=\lim _{h \rightarrow t ; h \in[a, b]} \frac{\gamma(h)-\gamma(t)}{h-t}
$$

whenever the limit exists. This is equivalent to state that all derivatives

$$
\gamma_{j}^{\prime}(t):=\lim _{h \rightarrow t ; h \in[a, b]} \frac{\gamma_{j}(h)-\gamma_{j}(t)}{h-t}, \quad j=1, \ldots, d,
$$

exist in $t$.

Definition 6. A path $\gamma:[a, b] \rightarrow \mathbb{R}^{d}$ is said to be smooth if it is a function of class $C^{1}([a, b])$ and $\gamma^{\prime}(t) \neq 0$ for any $t \in(a, b)$.

An additional piece of information related to the regularity of a path regards the evaluation of its arc length.

Definition 7. Let $\gamma:[a, b] \rightarrow \mathbb{R}^{d}$ be a path and let $\Pi$ the set of all finite partitions $\pi:=\left\{a=t_{1}<\cdots<t_{k}=b\right\}$ of the interval $[a, b]$. Denote by

$$
L(\gamma, \pi):=\sum_{i=1}^{k-1}\left\|\gamma\left(t_{i+1}\right)-\gamma\left(t_{i}\right)\right\| .
$$

The path $\gamma$ is said rectifiable or of bounded variation if

$$
L(\gamma):=\sup _{\pi \in \Pi} L(\gamma, \pi)<+\infty ;
$$

this quantity is referred to as the length of $\gamma$.

The following theorem holds [Galbis and Maestre 2012, p. 24].

Theorem 8. If $\gamma$ is a smooth curve whose domain is an interval $[a, b] \subset \mathbb{R}$, then it is rectifiable and its length is given by

$$
L(\gamma)=\int_{a}^{b}\left\|\gamma^{\prime}(t)\right\| \mathrm{d} t .
$$

Remark 9. The above theorem can be easily extended to a piecewise smooth path [Galbis and Maestre 2012, p. 26].

Example 10. The following are examples of smooth simple curves:

1. A segment in $\mathbb{R}^{d}$ with end points $x_{\text {ini }}$ and $x_{\text {fin }} \in \mathbb{R}^{d}$ :

$$
\Gamma=\left\{x_{\text {ini }} \cdot t+x_{\text {fin }} \cdot(1-t): t \in[0,1]\right\} .
$$


2. A circle in $\mathbb{R}^{2}$ :

$$
\Gamma=\{(\cos t, \sin t): t \in[0,2 \pi]\} .
$$

These sets are 1-regular, according to Definition 1.

If $\gamma:[a, b] \rightarrow \mathbb{R}^{d}$ is a smooth path, denote by $\Gamma:=\gamma([a, b]) \subset \mathbb{R}^{d}$ its support; we may associate to $\Gamma$ the Radon measure defined as

$$
\mu_{\Gamma}: A \in \mathcal{B}_{\mathbb{R}^{d}} \mapsto \mu_{\Gamma}(A):=\mathcal{H}^{1}(\Gamma \cap A) .
$$

The following theorem shows the action of the measure $\mu_{\Gamma}$ on test functions $f \in C_{c}\left(\mathbb{R}^{d}, \mathbb{R}^{d}\right)$ (see, e.g., [Evans and Gariepy 1992]).

Theorem 11. If $\gamma:[a, b] \rightarrow \mathbb{R}^{d}$ is a $C^{1}([a, b])$ simple curve such that $\Gamma=$ $\gamma([a, b])$, then for all $f \in C_{c}\left(\mathbb{R}^{d}, \mathbb{R}^{d}\right)$,

$$
\int f(x) \mu_{\Gamma} \mathrm{d} x=\int_{a}^{b} f(\gamma(t))\left\|\gamma^{\prime}(t)\right\| \mathrm{d} t .
$$

Similarly, for every $A \in \mathcal{B}\left(\mathbb{R}^{d}\right)$,

$$
\mu_{\Gamma}(A)=\int_{a}^{b} \epsilon_{\gamma(t)}(A)\left\|\gamma^{\prime}(t)\right\| \mathrm{d} t
$$

\subsection{The stochastic case.}

2.2.1. Random Radon measures. Consider the space $C_{c}\left(\mathbb{R}^{d}, \mathbb{R}\right)$ of continuous functions with compact support. This space is the union of the separable Banach spaces $C(K, \mathbb{R})$ over all compact sets $K \in \mathbb{R}^{d}$; let us write $\|\cdot\|_{K}$ for the norm on $C(K)$ :

$$
\|f\|_{K}=\sup _{x \in K}|f(x)| .
$$

The space $C_{c}\left(\mathbb{R}^{d}, \mathbb{R}\right)$ can be endowed with the direct limit topology associated to a family $C\left(K_{n}, \mathbb{R}\right)$ with $K_{n}$ increasing to $\mathbb{R}^{d}$; we do not need the details of this definition but we need to know the following useful fact: a linear functional $I: C_{c}\left(\mathbb{R}^{d}, \mathbb{R}\right) \rightarrow \mathbb{R}$ is continuous if and only if for every compact $K \in \mathbb{R}^{d}$ there is $C_{K}>0$ such that

$$
|I(f)| \leq C_{K}\|f\|_{K} \quad \text { for every } f \in C(K, \mathbb{R}) .
$$

Moreover, the dual of $C_{c}\left(\mathbb{R}^{d}, \mathbb{R}\right)$ is the space of signed Radon measures, which we shall denote by $\mathcal{M}$.

Definition 12. Given a probability space $(\Omega, \mathcal{F}, P)$, a random Radon measure is a map $\mu: \Omega \rightarrow \mathcal{M}$ such that for every $f \in C_{c}\left(\mathbb{R}^{d}, \mathbb{R}\right)$, the function $(\mu, f):=$ $\int_{\mathbb{R}^{d}} f(x) \mu \mathrm{d} x: \Omega \rightarrow \mathbb{R}$ is measurable. We shall write $\left(\mu_{\omega}, f\right)$ to emphasize the dependence on $\omega$ when $\mu$ is random. 
Proposition 13 (expected value of a random Radon measure). Let $\mu: \Omega \rightarrow \mathcal{M}$ be a random Radon measure. Assume that for every compact $K \in \mathbb{R}^{d}$ there is $C_{K}>0$ such that

$$
\int_{\Omega}\left|\left(\mu_{\omega}, f\right)\right| P \mathrm{~d} \omega \leq C_{K}\|f\|_{K},
$$

for every $f \in C(K, \mathbb{R})$. Then there exists an element $\hat{\mu} \in \mathcal{M}$ such that

$$
(\hat{\mu}, f)=\int_{\Omega}\left(\mu_{\omega}, f\right) P \mathrm{~d} \omega=\mathbb{E}[(\mu, f)],
$$

for every $f \in C_{c}\left(\mathbb{R}^{d}, \mathbb{R}\right)$.

Proof. Define the number $(\hat{\mu}, f)$ by the previous identity; for every $f \in C_{c}\left(\mathbb{R}^{d}, \mathbb{R}\right)$, since $f \in C(K, \mathbb{R})$ for some compact set $K$, we have $\int_{\Omega}\left|\left(\mu_{\omega}, f\right)\right| P \mathrm{~d} \omega<\infty$ by assumption, hence $\int_{\Omega}\left(\mu_{\omega}, f\right) P \mathrm{~d} \omega$ is well-defined.

Denote by $\Phi: f \in C_{c}\left(\mathbb{R}^{d}, \mathbb{R}\right) \mapsto \Phi(f):=(\mu, f) \in L^{1}(\Omega)$. We have to show that, as a function of $f$, the number $(\hat{\mu}, f)$ is linear continuous on $C_{c}\left(\mathbb{R}^{d}\right)$. Taking $f, g \in C_{c}\left(\mathbb{R}^{d}, \mathbb{R}\right)$ and $\alpha, \beta \in \mathbb{R}$, since $\Phi$ is linear, we have $\Phi_{\omega}(\alpha f+\beta g)=\alpha \Phi_{\omega}(f)+$ $\beta \Phi_{\omega}(g)$, hence

$$
\int_{\Omega} \Phi_{\omega}(\alpha f+\beta g) P \mathrm{~d} \omega=\alpha \int_{\Omega} \Phi_{\omega}(f) P \mathrm{~d} \omega+\beta \int_{\Omega} \Phi_{\omega}(g) P \mathrm{~d} \omega,
$$

which implies the linearity. For the continuity, for every compact set $K$ and $f \in$ $C(K, \mathbb{R})$, we have

$$
|(\hat{\mu}, f)| \leq \int_{\Omega}\left|\Phi_{\omega}(f)(\omega)\right| P \mathrm{~d} \omega \leq C_{K}\|f\|_{K}
$$

by assumption, hence $\hat{\mu}$ is a continuous functional.

Under the assumptions of Proposition 13, the measure $\hat{\mu} \in \mathcal{M}$, which satisfies the equality

$$
(\hat{\mu}, f)=\mathbb{E}[(\mu, f)]
$$

for every $f \in C_{c}\left(\mathbb{R}^{d}, \mathbb{R}\right)$ will be called the expected value of the measure $\mu$ and will be denoted by $\mathbb{E}[\mu]$.

2.2.2. Random linear functionals. Let $S$ be a separable linear metric space of test functions, for instance, $C_{c}\left(\mathbb{R}^{d}, \mathbb{R}\right)$ or $C_{c}^{\infty}\left(\mathbb{R}^{d}, \mathbb{R}\right)$ or $L^{2}(0, T)$. Denote by $S^{\prime}$ the space of continuous linear functionals on $S$. Let $(\Omega, \mathcal{F}, P)$ be a probability space. The following definition generalizes Definition 12.

Definition 14. We call random functional in the strict sense any map $\phi: \Omega \rightarrow S^{\prime}$ such that $(\phi, f)$ is measurable for every $f \in S$. 
Remark 15. The definition above is analogous to the well-known definition for Banach valued random variables (see, e.g., [Araujo and Giné 1980; Bharucha-Reid 1972; Bosq 2000]).

Later we will consider random functionals in a broad sense.

2.3. Random functionals associated with random closed sets. We recall that a random closed set $\Xi$ in $\mathbb{R}^{d}$ is a measurable map

$$
\Xi:(\Omega, \mathcal{F}, \mathbb{P}) \longrightarrow\left(\mathbb{F}, \sigma_{\mathbb{F}}\right),
$$

where $\mathbb{F}$ denotes the class of the closed subsets in $\mathbb{R}^{d}$, and $\sigma_{\mathbb{F}}$ is the $\sigma$-algebra generated by the so-called hit-or-miss topology (see [Matheron 1975]).

Definition 16. Given an integer $n$ with $0 \leq n \leq d$, we say that a random closed set $\Theta$ in $\mathbb{R}^{d}$ is $n$-regular if it satisfies the following conditions:

(i) for almost all $\omega \in \Omega$, the set $\Theta(\omega)$ is an $n$-regular closed set in $\mathbb{R}^{d}$; and

(ii) $\mathbb{E}\left[\mathcal{H}^{n}\left(\Theta \cap B_{R}(0)\right)\right]<\infty$ for any $R>0$.

Suppose now that $\Theta$ is a random $n$-regular closed set in $\mathbb{R}^{d}$. Thanks to the assumptions on the random set $\Theta$, it can be shown that the random measure $\mu_{\Theta}$ is $P$-a.s. a Radon measure on $\mathcal{B}_{\mathbb{R}^{d}}$; it can be further shown that, as a map $\mu_{\Theta}: \omega \in$ $\Omega \mapsto \mu_{\Theta(\omega)} \in \mathcal{M}$, it is a random Radon measure according to Definition 12 (see, e.g., [Baddeley and Molchanov 1997; Matheron 1965; Zähle 1982]).

In this case it makes sense to define, for any $\omega \in \Omega$, the linear functional $\delta_{\Theta(\omega)}$ such that, for any $f \in C_{c}\left(\mathbb{R}^{d}, \mathbb{R}\right)$ :

$$
\left(\delta_{\Theta}, f\right):=\int_{\mathbb{R}^{d}} f(x) \mu_{\Theta}(x) \mathrm{d} x, \quad P \text {-a.s. }
$$

According to Definition $14, \delta_{\Theta}$ is then a random linear functional in the strict sense.

By recollecting all of the above, we may finally state the following.

Proposition 17. Let $\Theta$ be a random closed n-regular set in $\mathbb{R}^{d}$. Then the associated random Radon measure $\mu_{\Theta}$ satisfies the assumption of Proposition 13, and therefore the expected value $\mathbb{E}\left[\mu_{\Theta}\right]$ is well-defined. We therefore define the expected value of the generalized density $\delta_{\Theta}$ by the following identities:

$$
\begin{aligned}
\left(\mathbb{E}\left[\delta_{\Theta}\right], f\right) & =\int_{\mathbb{R}^{d}} f(x) \mathbb{E}\left[\delta_{\Theta}\right](x) \mathrm{d} x \\
& :=\int_{\mathbb{R}^{d}} f(x) \mathbb{E}\left[\mu_{\Theta}\right] \mathrm{d} x=\mathbb{E}\left[\int_{\mathbb{R}^{d}} f(x) \mu_{\Theta}(x) \mathrm{d} x\right] \\
& =\mathbb{E}\left[\int_{\mathbb{R}^{d}} f(x) \delta_{\Theta} \mathrm{d} x\right]=\mathbb{E}\left[\left(\delta_{\Theta}, f\right)\right],
\end{aligned}
$$

for any $f \in C_{c}\left(\mathbb{R}^{d}, \mathbb{R}\right)$. 
Proof. Let $K$ be a compact in $\mathbb{R}^{d}$ and $f \in C_{c}(K, \mathbb{R})$. We have

$$
\begin{aligned}
\int_{\Omega}\left|\left(\mu_{\Theta(\omega)}, f\right)\right| P \mathrm{~d} \omega & =\int_{\Omega}\left|\int_{\mathbb{R}^{d}} f(x) \mu_{\Theta(\omega)} \mathrm{d} x\right| P \mathrm{~d} \omega \\
& \leq \int_{\Omega} \int_{\mathbb{R}^{d}}|f(x)| \mu_{\Theta(\omega)} \mathrm{d} x P \mathrm{~d} \omega \\
& =\int_{\Omega} \int_{K}|f(x)| \mu_{\Theta(\omega)} \mathrm{d} x P \mathrm{~d} \omega,
\end{aligned}
$$

because $f$ has support in $K$, and

$$
\begin{aligned}
\int_{\Omega}\left|\left(\mu_{\Theta(\omega)}, f\right)\right| P \mathrm{~d} \omega & \leq\|f\|_{K} \int_{\Omega} \int_{K} \mu_{\Theta(\omega)} \mathrm{d} x P \mathrm{~d} \omega \\
& =\|f\|_{K} \int_{\Omega} \mu_{\Theta(\omega)}(K) P \mathrm{~d} \omega \\
& =\|f\|_{K} \mathbb{E}\left[\mathcal{H}^{n}(\Theta \cap K)\right] \\
& \leq C_{K}\|f\|_{K},
\end{aligned}
$$

by (ii) of the previous definition (the set $K$ is included in a ball $B_{R}(0)$ ). Hence we may apply Proposition 13 for the existence of the expected value $\mathbb{E}\left[\mu_{\Theta}\right]$.

2.3.1. Absolutely continuous (in mean) random sets.

Remark 18. When $n=d$, the integral and expectation in (2-9) can be exchanged by Fubini's theorem, since in this case both $\mu_{\Theta}$ and $\mathbb{E}\left[\mu_{\Theta}\right]$ are absolutely continuous with respect to $v^{d}$ and $\delta_{\Theta}(x)=\mathbb{1}_{\Theta}(x), v^{d}$-a.s.

In particular, $\delta_{\Theta}(x)=\mathbb{1}_{\Theta}(x), v^{d}$-a.s. implies that

$$
\mathbb{E}\left[\delta_{\Theta}\right](x)=\mathbb{P}(x \in \Theta), \quad v^{d} \text {-a.s. }
$$

and the following chain of equalities are well-known (according to our definition of $\mathbb{E}\left[\delta_{\Theta}\right][$ Kolmogorov 1956, p. 46]):

$$
\begin{aligned}
\mathbb{E}\left[v^{d}(\Theta \cap A)\right] & =\mathbb{E}\left(\int_{\mathbb{R}^{d}} \mathbf{1}_{\Theta \cap A}(x) \mathrm{d} x\right)=\mathbb{E}\left(\int_{A} \mathbf{1}_{\Theta}(x) \mathrm{d} x\right) \\
& =\int_{A} \mathbb{E}\left(\mathbf{1}_{\Theta}(x)\right) \mathrm{d} x=\int_{A} \mathbb{P}(x \in \Theta) \mathrm{d} x .
\end{aligned}
$$

In materials science, the density

$$
\rho(x):=\mathbb{E}\left[\delta_{\Theta}\right](x)=\mathbb{P}(x \in \Theta)
$$

is known as the (degree of) crystallinity.

If $\Theta$ is not a pathological set, i.e., if $\mathcal{H}^{n}(\Theta)(\omega)>0$ with $\mathbb{P}$-a.e. for $\omega \in \Omega(n<d)$, we may notice that, even though for a.e. realization $\Theta$ of $\Theta$ the measure $\mu_{\Theta}$ is 
positive and singular (and so it is not absolutely continuous), and the expected measure $\mathbb{E}\left[\mu_{\Theta}\right]$ may be absolutely continuous with respect to $v^{d}$.

Example. Consider the case $n=0$. Let $\Theta=X_{0}$ be a random point in $\mathbb{R}^{d}$; in this case, $\mathcal{H}^{0}\left(X_{0} \cap A\right)=\mathbb{1}_{A}\left(X_{0}\right)$, and so

$$
\mathbb{E}\left[\mathcal{H}^{0}\left(X_{0} \cap A\right)\right]=\mathbb{P}\left(X_{0} \in A\right) .
$$

If $X_{0}$ is a continuous random point with the pdf $p_{X_{0}}$, then $\mathbb{E}\left[\mathcal{H}^{0}\left(X_{0} \cap \cdot\right)\right]$ is absolutely continuous and, in this case, $\mathbb{E}\left[\delta_{X_{0}}\right](x)$ is just the probability density function $p_{X_{0}}(x)$, so $\int_{A} \mathbb{E}\left[\delta_{X_{0}}\right](x) v^{d} \mathrm{~d} x$ is the usual Lebesgue integral. Note that we formally have

$$
\mathbb{E}\left[\delta_{X_{0}}\right](x)=\int_{\mathbb{R}^{d}} \delta_{y}(x) p_{X_{0}}(y) v^{d} \mathrm{~d} y=\int_{\mathbb{R}^{d}} \delta_{x}(y) p_{X_{0}}(y) v^{d} \mathrm{~d} y=p_{X_{0}}(x) ;
$$

and in accordance with Proposition 17,

$$
\begin{aligned}
\int_{A} \mathbb{E}\left[\delta_{X_{0}}\right](x) v^{d} \mathrm{~d} x & =\int_{A} p_{X_{0}}(x) v^{d} \mathrm{~d} x=\mathbb{P}\left(X_{0} \in A\right) \\
& =\mathbb{E}\left[\mathcal{H}^{0}\left(X_{0} \cap A\right)\right]=\mathbb{E}\left[\int_{A} \delta_{X_{0}}(x) v^{d} \mathrm{~d} x\right] .
\end{aligned}
$$

If $X_{0}$ is discrete, i.e., $X_{0}=x_{i}$ with probability $p_{i}$, only for an at most countable set of points $x_{i} \in \mathbb{R}^{d}$, then $\mathbb{E}\left[\mathcal{H}^{0}\left(X_{0} \cap \cdot\right)\right]$ is singular and, as in the previous case, we have that $\mathbb{E}\left[\delta_{X_{0}}\right](x)$ coincides with the probability distribution $p_{X_{0}}$ of $X_{0}$.

In fact, in this case $p_{X_{0}}(x)=\sum_{i} p_{i} \delta_{x_{i}}(x)$, and by computing the expectation of $\delta_{X_{0}}$, we formally obtain

$$
\mathbb{E}\left[\delta_{X_{0}}\right](x)=\delta_{x_{1}}(x) p_{1}+\delta_{x_{2}}(x) p_{2}+\cdots=\sum_{i} p_{i} \delta_{x_{i}}(x)=p_{X_{0}}(x) .
$$

Remark 19. By Remark 18 and the considerations in the above example, we may claim that, in the cases $n=d$ and $n=0$ with $X_{0}$ being continuous, the expected linear functionals $\mathbb{E}\left[\delta_{\Theta}\right]$ and $\mathbb{E}\left[\delta_{X_{0}}\right]$ are defined by the function $\rho(x):=\mathbb{P}(x \in \Theta)$ and by the pdf $p_{X_{0}}$ of $X_{0}$, respectively, in the following way:

$$
\left(\mathbb{E}\left[\delta_{\Theta}\right], f\right):=\int_{\mathbb{R}^{d}} f(x) \rho(x) \mathrm{d} x
$$

and

$$
\left(\mathbb{E}\left[\delta_{X_{0}}\right], f\right):=\int_{\mathbb{R}^{d}} f(x) p_{X_{0}}(x) \mathrm{d} x .
$$

In fact, let us consider the random point $X_{0}$; in accordance with Proposition 17,

$$
\left(\mathbb{E}\left[\delta_{X_{0}}\right], f\right):=\int_{\mathbb{R}^{d}} f(x) p_{X_{0}}(x) \mathrm{d} x=\mathbb{E}\left[f\left(X_{0}\right)\right]=\mathbb{E}\left[\left(\delta_{X_{0}}, f\right)\right] .
$$


Of particular interest is the case of fiber processes, i.e., 1-dimensional random sets that occur in medicine as models for vessels, nerves, etc., and in materials science as models for line dislocations (defects in a crystalline material) [Hochrainer et al. 2007], etc. In these cases, an empirical definition of line density is given as the total length of dislocation lines per unit volume (see, e.g., [Callister Jr. 2007, p. 177]). This has given a strong motivation for a rigorous definition of the mean density of random sets of any Hausdorff dimension; for a discussion about continuity and absolute continuity of random closed sets, we refer to [Capasso and Villa 2006; 2007].

2.3.2. Example: an absolutely continuous 1-regular random set. Let $\left(X_{t}, V_{t}\right)$ be the solution of the stochastic differential system in $\mathbb{R}^{d}$ :

$$
\begin{aligned}
\mathrm{d} X_{t} & =V_{t} \mathrm{~d} t, \\
\mathrm{~d} V_{t} & =b\left(X_{t}, V_{t}\right) \mathrm{d} t+\mathrm{d} W_{t},
\end{aligned}
$$

where $b$ is Lipschitz continuous and $W_{t}$ is a Brownian motion in $\mathbb{R}^{d}$. If the initial condition $\left(X_{0}, V_{0}\right)$ of the above system has a smooth density $p_{0}(x, v)$ with respect to the usual Lebesgue measure on $\mathbb{R}^{d}$, thanks to hypoellipticity, $\left(X_{t}, V_{t}\right)$ has (for every $t>0)$ a smooth density $p_{t}(x, v)$ with respect to the Lebesgue measure; it is a solution to the Fokker-Planck equation:

$$
\begin{aligned}
\frac{\partial p}{\partial t} & =\Delta_{v} p-v \cdot \nabla_{x} p-\operatorname{div}_{v}(b p), \\
\left.p\right|_{t=0} & =p_{0} .
\end{aligned}
$$

Given $T>0$, consider the random path in $\mathbb{R}^{d}$ given by $t \in[0, T] \mapsto X_{t} \in \mathbb{R}^{d}$ (not necessarily simple); in accordance with Theorem 11, the associated random Radon measure $\mu_{X}$ is such that

$$
\int f(x) \mu_{X} \mathrm{~d} x=\int_{0}^{T} f\left(X_{t}\right)\left\|V_{t}\right\| \mathrm{d} t, \quad f \in C_{c}\left(\mathbb{R}^{d}, \mathbb{R}\right) .
$$

This formula defines a random generalized density $\delta_{X}(x)$ by the identity

$$
\left(\delta_{X}, f\right)=\int_{0}^{T} f\left(X_{t}\right)\left\|V_{t}\right\| \mathrm{d} t, \quad f \in C_{c}\left(\mathbb{R}^{d}, \mathbb{R}\right) .
$$

It can be checked that we have

$$
\delta_{X}(x)=\int_{0}^{T} \delta_{X_{t}}(x)\left\|V_{t}\right\| \mathrm{d} t=\int_{0}^{T} \delta_{X_{t}}(x)\left\|X_{t}^{\prime}\right\| \mathrm{d} t .
$$

Proposition 20. Assume $\mathbb{E}\left[\left\|X_{0}\right\|+\left\|V_{0}\right\|\right]<\infty$. Then the random generalized density $\delta_{X}$, which is a.s. concentrated on the random curve $X$, has a smooth average 
density $\mathbb{E}\left[\delta_{X}\right](x)$ given by

$$
\mathbb{E}\left[\delta_{X}\right](x)=\int_{0}^{T} \int_{\mathbb{R}^{d}}\|v\| p_{t}(x, v) \mathrm{d} v \mathrm{~d} t .
$$

Proof. We only sketch the proof. We have, with $\Theta_{1}$ equal to the support of the curve $X$,

$$
\begin{aligned}
\mathbb{E}\left[\mathcal{H}^{1}\left(\Theta_{1} \cap B_{R}(0)\right)\right] & =\mathbb{E}\left[\int_{0}^{T} 1_{B_{R}(0)}\left(X_{t}\right)\left\|V_{t}\right\| \mathrm{d} t\right] \\
& \leq \mathbb{E}\left[\int_{0}^{T}\left\|V_{t}\right\| \mathrm{d} t\right]=\int_{0}^{T} \mathbb{E}\left[\left\|V_{t}\right\|\right] \mathrm{d} t .
\end{aligned}
$$

We continue the proof under the additional assumption that $b$ is bounded, otherwise it is sufficient to use a Gronwall-type argument (it is here that we use $\mathbb{E}\left[\left\|X_{0}\right\|\right]<\infty$ ). We have

$$
V_{t}=V_{0}+\int_{0}^{t} b\left(X_{s}, V_{s}\right) \mathrm{d} s+W_{t}
$$

hence

$$
\left\|V_{t}\right\| \leq\left\|V_{0}\right\|+T\|b\|_{\infty}+\left\|W_{t}\right\|
$$

which implies $\sup _{t \in[0, T]} \mathbb{E}\left[\left\|V_{t}\right\|\right]<\infty$ (because $\mathbb{E}\left[\left\|V_{0}\right\|\right]<\infty$ ), hence

$$
\int_{0}^{T} \mathbb{E}\left[\left\|V_{t}\right\|\right] \mathrm{d} t<\infty
$$

and therefore $\mathbb{E}\left[\mathcal{H}^{1}\left(\Theta_{1} \cap B_{R}(0)\right)\right]<\infty$. By the general criterion above, this implies that $\mathbb{E}\left[\delta_{X}\right]$ exists and

$$
\left(\mathbb{E}\left[\delta_{X}\right], f\right)=\int_{0}^{T} \mathbb{E}\left[f\left(X_{t}\right)\left\|V_{t}\right\|\right] \mathrm{d} t .
$$

It follows that

$$
\begin{aligned}
\left(\mathbb{E}\left[\delta_{X}\right], f\right) & =\int_{0}^{T} \int_{\mathbb{R}^{d}} \int_{\mathbb{R}^{d}} f(x)\|v\| p_{t}(x, v) \mathrm{d} x \mathrm{~d} v \mathrm{~d} t \\
& =\int_{\mathbb{R}^{d}}\left(\int_{0}^{T} \int_{\mathbb{R}^{d}}\|v\| p_{t}(x, v) \mathrm{d} v \mathrm{~d} t\right) f(x) \mathrm{d} x .
\end{aligned}
$$

The arbitrarity of $f$ gives us the existence of the average density $\mathbb{E}\left[\delta_{X}\right](x)$ and its formula.

Hence we may claim that the path $X$ is a 1-dimensional absolutely continuous (in mean) random set in $\mathbb{R}^{d}$. 
2.4. More on random functionals. Given a probability space $(\Omega, \mathcal{F}, P)$, we denote by $L^{0}(\Omega)$ the space of $P$-equivalence classes of random variables $X: \Omega \rightarrow \mathbb{R}$, endowed with the Ky Fan topology, i.e., the topology of the convergence in probability.

In the previous sections, we defined as random functionals all measurable mappings from $\Omega$ to the dual space $C_{c}\left(\mathbb{R}^{d}, \mathbb{R}\right)^{\prime}$ (we continue to restrict ourselves to functionals on $C_{c}\left(\mathbb{R}^{d}, \mathbb{R}\right)$ ). However, there exist examples of random objects which are natural to call random functionals, but do not enter in the previous definition. Let us then introduce a broader concept.

Definition 21. We use random functional in the broad sense of any continuous linear map $\Phi: C_{c}\left(\mathbb{R}^{d}, \mathbb{R}\right) \rightarrow L^{0}(\Omega)$. When there exists a random functional in the strict sense $\phi: \Omega \rightarrow C_{c}\left(\mathbb{R}^{d}, \mathbb{R}\right)^{\prime}$ such that

$$
\Phi(f)(\omega)=\left(\phi_{\omega}, f\right)
$$

for all $f \in C_{c}\left(\mathbb{R}^{d}, \mathbb{R}\right)$ and for a.a. $\omega \in \Omega$, we say that $\phi_{\omega}$ is a pathwise realization of $\Phi$.

Example 22. Let $W$ be a Brownian motion on $(\Omega, \mathcal{F}, P)$ and let $d=1$. To any function $f \in C_{c}(\mathbb{R}, \mathbb{R})$, we associate the random variable

$$
\Phi(f)=\int_{0}^{T} f\left(W_{t}\right) \mathrm{d} W_{t} .
$$

It is known that there is no simple pathwise meaning for this integral: we cannot fix $\omega \in \Omega$ and consider it as a map from $C_{c}(\mathbb{R}, \mathbb{R})$ to $\mathbb{R}$. This is an example of a random functional in the broad sense which has no pathwise realization.

Example 23. Referring to the above example, a pathwise realization exists if we restrict ourselves to more regular test functions $f$. Indeed, if $f \in C_{c}^{1}(\mathbb{R}, \mathbb{R})$, and $F^{\prime}=f$, by Itô's formula we have

$$
F\left(W_{T}\right)=F\left(W_{0}\right)+\int_{0}^{T} f\left(W_{t}\right) \mathrm{d} W_{t}+\frac{1}{2} \int_{0}^{T} f^{\prime}\left(W_{t}\right) \mathrm{d} t,
$$

and therefore the random functional in the strict sense $\phi_{\omega}$, defined as

$$
\left(\phi_{\omega}, f\right)=F\left(W_{T}(\omega)\right)-F\left(W_{0}(\omega)\right)-\frac{1}{2} \int_{0}^{T} f^{\prime}\left(W_{t}(\omega)\right) \mathrm{d} t
$$

(these expressions are all well-defined path by path) satisfies

$$
(\phi ., f)=\int_{0}^{T} f\left(W_{t}\right) \mathrm{d} W_{t}=\Phi(f), \quad P \text {-a.s. }
$$

hence $\phi_{\omega}$ is a pathwise realization of $\Phi$; but only on $f \in C_{c}^{1}(\mathbb{R}, \mathbb{R})$. 
Example 24. If we allow ourselves to use $L^{2}(0, T)$ as a set of test functions in place of $C_{c}\left(\mathbb{R}^{d}, \mathbb{R}\right)$, the classical Wiener integral

$$
\Phi(f)=\int_{0}^{T} f_{t} \mathrm{~d} W_{t}
$$

is another example of random functional in the broad sense which has no pathwise realization. Again, if we change the set of test functions and we take $f \in$ $W^{1,2}(0, T)$, the pathwise realization exists, given by

$$
\left(\phi_{\omega}, f\right)=f_{T} W_{T}-\int_{0}^{T} f_{t}^{\prime} W_{t} \mathrm{~d} t .
$$

The previous examples have mainly an academic character, so we do not insist on this notion here. However, in the context of random currents, there are examples of great potential interest for applications, so we shall enter in more detail.

\section{Currents}

In this section we wish to extend our analysis to vector fields in $\mathbb{R}^{d}$, hence to random currents. Motivations for the study of random currents are the same as anticipated in Section 2.3 for 1-dimensional random sets, i.e., fiber processes.

We will start by defining line integrals of differential forms (see, e.g., [Giaquinta et al. 1998; Giaquinta and Modica 2009; Galbis and Maestre 2012, p. 21; Buck 1956]).

3.1. Differential forms. Let $g: U \subset \mathbb{R}^{d} \rightarrow \mathbb{R}$ be a real function of class $C^{1}$ in the open set $U$, i.e., it has all partial derivatives of the first order continuous in $U$. The differential of $g$ at a point $x \in U$ is the linear function $\operatorname{dg}(x): \mathbb{R}^{d} \rightarrow \mathbb{R}$ such that

$$
h=\left(h_{1}, \ldots, h_{d}\right) \in \mathbb{R}^{d} \mapsto \operatorname{dg}(x)(h)=\sum_{j=1}^{d} \frac{\partial g}{\partial x_{j}}(x) h_{j} .
$$

It is usually suggestive to denote the partial increments $h_{j}=\left\langle e_{j}, h\right\rangle$ by $\mathrm{d} x_{j}$, so that the expression (3-1) is usually written as

$$
\mathrm{d} x=\left(\mathrm{d} x_{1}, \ldots, \mathrm{d} x_{d}\right) \in \mathbb{R}^{d} \mapsto \operatorname{dg}(x)(\mathrm{d} x)=\sum_{j=1}^{d} \frac{\partial g}{\partial x_{j}}(x) \mathrm{d} x_{j} .
$$

Expressions (3-1) or (3-2) are a particular case of a more general mathematical entity called differential form of degree one, or simply 1-form, defined below.

Definition 25. Let $U \subset \mathbb{R}^{d}$ be an open set. A differential form of degree one or simply a 1 -form on $U$ is a mapping

$$
\omega: U \rightarrow \mathcal{L}\left(\mathbb{R}^{d}, \mathbb{R}\right)=\left(\mathbb{R}^{d}\right)^{\prime},
$$


where $\mathcal{L}\left(\mathbb{R}^{d}, \mathbb{R}\right)$ denotes the space of all real valued linear functions on $\mathbb{R}^{d}$, i.e., the dual space $\left(\mathbb{R}^{d}\right)^{\prime}$ of $\mathbb{R}^{d}$.

Given a 1 -form $\omega$, a point $x \in U$, and an integer $j=1, \ldots, d$, we will denote the scalar $\omega(x)\left(e_{j}\right) \in \mathbb{R}$ by $f_{j}(x)$. Evidently $f_{j}: U \rightarrow \mathbb{R}$; by the linearity of $\omega(x) \in\left(\mathbb{R}^{d}\right)^{\prime}$, for any $h=\left(h_{1}, \ldots, h_{d}\right) \in \mathbb{R}^{d}$ we have

$$
\omega(x)(h)=\sum_{j=1}^{d} f_{j}(x) h_{j},
$$

or

$$
\omega(x)(\mathrm{d} x)=\sum_{j=1}^{d} f_{j}(x) \mathrm{d} x_{j} .
$$

This expression is usually abbreviated into

$$
\omega=\sum_{j=1}^{d} f_{j} \mathrm{~d} x_{j} .
$$

The functions $f_{j}$, where $j=1, \ldots d$, are called the component functions of the differential form $\omega$. We will usually assume that the component functions of a 1 -form are continuous; in such a case we say that the form is continuous.

Remark 26. The above discussion shows that the study of a 1-form is essentially equivalent to the study of a vector field, say

$$
F(x)=\left(f_{1}(x), \ldots, f_{d}(x)\right) ; \quad x \in U .
$$

3.2. Line integrals of differential forms. The line integral of a differential form was originally motivated by the calculation of the work done by a force field along a path.

Consider a smooth path $\gamma:[a, b] \rightarrow \mathbb{R}^{3}$ all contained in an open set $U \subset \mathbb{R}^{3}$; and let $F: U \rightarrow \mathbb{R}^{3}$ be a force field acting on a point object. The work done by the force field $F$ along the path $\gamma$ can be obtained by taking into account two facts:

1. The work done by a force field along a path depends only upon the component of the force along the tangent direction of the path at each point.

2. The work done by a constant field $F_{0}$ to move an object along a line segment in its direction is given by the product of $\left\|F_{0}\right\|$ and the length of the line segment.

Consider a very fine partition $\pi=\left\{a=t_{1}<\cdots<t_{k}=b\right\}$ of the interval $[a, b]$, so that the arc length of $\left.\gamma\right|_{\left[t_{j}, t_{j+1}\right]}$, according to Theorem 8 , can be approximated by

$$
\left\|\gamma^{\prime}\left(t_{j}\right)\right\|\left(t_{j+1}-t_{j}\right) \text {. }
$$


We know that the unit vector which is tangent to a smooth path at $\gamma(t), t \in[a, b]$ is given by

$$
T(t)=\frac{\gamma^{\prime}(t)}{\left\|\gamma^{\prime}(t)\right\|},
$$

so that we may assume, at a good approximation, that along the path $\left.\gamma\right|_{\left[t_{j}, t_{j+1}\right]}$ the force field has a constant value $F\left(\gamma\left(t_{j}\right)\right)$. Consequently, the work done by the force field $F$ along $\left.\gamma\right|_{\left[t_{j}, t_{j+1}\right]}$ can be approximated by

$$
\left\langle F\left(\gamma\left(t_{j}\right)\right), T\left(t_{j}\right)\right\rangle \| \gamma^{\prime}\left(t_{j} \|\left(t_{j+1}-t_{j}\right)=\left\langle F\left(\gamma\left(t_{j}\right)\right), \gamma^{\prime}\left(t_{j}\right)\right\rangle\left(t_{j+1}-t_{j}\right),\right.
$$

where $j=1, \ldots, k-1$. Summing up a good approximation of the work done in moving the object along $\gamma$ is given by

$$
\sum_{k=1}^{k-1}\left\langle F\left(\gamma\left(t_{j}\right)\right), \gamma^{\prime}\left(t_{j}\right)\right\rangle\left(t_{j+1}-t_{j}\right) ;
$$

the usual theorem of existence of the Riemann integral of a continuous function along a finite interval leads to the following proposition.

Proposition 27. The work done by a continuous force field $F: U \rightarrow \mathbb{R}^{3}$ along a piecewise smooth path $\gamma:[a, b] \rightarrow \mathbb{R}^{3}$ all contained in an open set $U \subset \mathbb{R}^{3}$ is given by

$$
\int_{\gamma} F=\int_{a}^{b}\left\langle F(\gamma(t)), \gamma^{\prime}(t)\right\rangle \mathrm{d} t
$$

The following definition is then meaningful.

Definition 28. Let $F: U \rightarrow \mathbb{R}^{d}$ be a continuous vector field along a piecewise smooth path $\gamma:[a, b] \rightarrow \mathbb{R}^{d}$ all contained in an open set $U \subset \mathbb{R}^{d}$. The line integral of $F$ along $\gamma$ is given by

$$
\int_{\gamma} F:=\int_{a}^{b}\left\langle F(\gamma(t)), \gamma^{\prime}(t)\right\rangle \mathrm{d} t
$$

It is clear that the line integral of a vector field depends upon the orientation of the path, i.e.,

$$
\int_{\gamma[a, b]} F:=-\int_{\gamma[b, a]} F .
$$

Thanks to the correspondence between 1-forms and vector fields, the following definition is appropriate.

Definition 29. Let $U \subset \mathbb{R}^{d}$ be an open set, let $\omega$ be a continuous 1 -form on $U$, and let $\gamma:[a, b] \rightarrow \mathbb{R}^{d}$ be a piecewise smooth path, all contained in $U$. If $F(x)=$ 
$\left(f_{1}(x), \ldots, f_{d}(x)\right)$, for $x \in U$, is the vector field associated with $\omega$, the line integral of $\omega$ along $\gamma$ is given by

$$
\begin{aligned}
\int_{\gamma} \omega:=\int_{a}^{b} \omega(\gamma(t)) \gamma^{\prime}(t) \mathrm{d} t & =\int_{a}^{b}\left\langle F(\gamma(t)), \gamma^{\prime}(t)\right\rangle \mathrm{d} t \\
& =\sum_{j=1}^{d} \int_{a}^{b} f_{j}(\gamma(t)) \gamma_{j}^{\prime}(t) \mathrm{d} t .
\end{aligned}
$$

3.3. 1-currents. In the sequel, a 1-current will be a continuous linear functional on $C_{c}\left(\mathbb{R}^{d}, \mathbb{R}^{d}\right)$ (more generally the literature considers a continuous linear functional on $\left.C_{c}^{\infty}\left(\mathbb{R}^{d}, \mathbb{R}^{d}\right)\right)$.

Typical examples of 1-currents are those induced by regular curves in $\mathbb{R}^{d}$. Let $\gamma:[a, b] \subset \mathbb{R} \rightarrow \mathbb{R}^{d}$ be a smooth curve in $\mathbb{R}^{d}$; the linear functional $\xi$ defined by

$$
\begin{aligned}
\theta \in C_{c}\left(\mathbb{R}^{d}, \mathbb{R}^{d}\right) \mapsto(\xi, \theta) & =\int_{a}^{b}\left\langle\theta(\gamma(t)), \gamma^{\prime}(t)\right\rangle \mathrm{d} t \\
& =\sum_{j=1}^{d} \int_{a}^{b} \theta_{j}(\gamma(t)) \gamma_{j}^{\prime}(t) \mathrm{d} t \in \mathbb{R}
\end{aligned}
$$

is a 1 -current.

Another case is one in which the 1-current is induced by a vector field. By an abuse of notation, let $\xi: \mathbb{R}^{d} \rightarrow \mathbb{R}^{d}$ be a vector field; the associated current is

$$
\theta \in C_{c}\left(\mathbb{R}^{d}, \mathbb{R}^{d}\right) \mapsto(\xi, \theta)=\int_{\mathbb{R}^{d}}\langle\theta(x), \xi(x)\rangle \mathrm{d} x=\sum_{j=1}^{d} \int_{\mathbb{R}^{d}} \theta_{j}(x) \xi_{j}(x) \mathrm{d} x .
$$

We may recover the case (3-10) as a particular case of (3-11) by the localization on the regular path $\{\gamma(t), t \in[a, b]\}$ made by a usual Dirac delta distribution

$$
\xi(x)=\int_{a}^{b} \delta_{\gamma(t)}(x) \gamma^{\prime}(t) \mathrm{d} t, \quad x \in \mathbb{R}^{d} ;
$$

i.e.,

$$
\xi=\int_{a}^{b} \delta_{\gamma(t)}(\cdot) \gamma^{\prime}(t) \mathrm{d} t
$$

3.4. Random 1-currents and their mean densities. We have said above that a deterministic 1-current is a linear continuous functional on $C_{c}^{\infty}\left(\mathbb{R}^{d}, \mathbb{R}^{d}\right)$. Since it is sufficient for our purposes, in order to stress the analogy with Radon measures, we consider afterwards only 1-currents which are linear continuous functionals on $C_{c}\left(\mathbb{R}^{d}, \mathbb{R}^{d}\right)$. There are two definitions of random 1-currents, as in the case of random functionals (see Section 2.2.2).

The most natural one is the following [Flandoli et al. 2009; Bessaih et al. 2017]. 
Definition 30. Given a probability space $(\Omega, \mathcal{F}, P)$, consider the space $C_{c}\left(\mathbb{R}^{d}, \mathbb{R}^{d}\right)$ of compact support continuous vector fields endowed with the topology of convergence on compact sets and let $C_{c}\left(\mathbb{R}^{d}, \mathbb{R}^{d}\right)^{\prime}$ be the space of 1-currents on $\mathbb{R}^{d}$, endowed with the dual topology, and the corresponding Borel sigma algebra. We call a random 1-current in the strict sense any measurable map

$$
\xi: \omega \in \Omega \mapsto \xi_{\omega} \in C_{c}\left(\mathbb{R}^{d}, \mathbb{R}^{d}\right)^{\prime} .
$$

The second, a weaker one, relates to the real value obtained when applying the current to a test vector field.

Definition 31. Given a probability space $(\Omega, \mathcal{F}, P)$, consider the space $L^{0}(\Omega)$ of real-valued random variables endowed with the Ky Fan topology, i.e., the topology of the convergence in probability, and the corresponding Borel sigma algebra. We call a random 1-current in the broad sense any continuous linear map $\Xi: C_{c}\left(\mathbb{R}^{d}, \mathbb{R}^{d}\right) \rightarrow L^{0}(\Omega)$. When there exists a random 1-current in the strict sense $\xi: \Omega \rightarrow C_{c}\left(\mathbb{R}^{d}, \mathbb{R}\right)^{\prime}$ such that

$$
\Xi(f)(\omega)=\left(\xi_{\omega}, f\right)
$$

for all $f \in C_{c}\left(\mathbb{R}^{d}, \mathbb{R}^{d}\right)$ and a.a. $\omega \in \Omega$, we say $\xi_{\omega}$ is a pathwise realization of $\Xi$.

One can show, as in the case of random functionals, that strict sense implies broad sense, but the opposite is not true. Below we provide a typical example for each category and compute the corresponding mean densities.

3.4.1. The mean of a 1-current. Consider a random 1-current in the strict sense $\xi_{\omega} \in C_{c}\left(\mathbb{R}^{d}, \mathbb{R}^{d}\right)^{\prime}$, and $\omega \in \Omega$. We say that it admits a mean value if a 1-current $\hat{\xi} \in C_{c}\left(\mathbb{R}^{d}, \mathbb{R}^{d}\right)^{\prime}$ exists such that, for any $\theta \in C_{c}\left(\mathbb{R}^{d}, \mathbb{R}^{d}\right)$,

$$
(\hat{\xi}, \theta)=\mathbb{E}[(\xi, \theta)]
$$

Whenever this happens, we will identify the current $\mathbb{E}[\xi]:=\hat{\xi}$ as the mean value of the current $\xi$.

We will say that the mean current $\mathbb{E}[\xi]$ is induced by a vector field if a locally integrable vector field $u: \mathbb{R}^{d} \rightarrow \mathbb{R}^{d}$ exists such that

$$
(\mathbb{E}[\xi], \theta)=\int_{\mathbb{R}^{d}}\langle\theta(x), u(x)\rangle \mathrm{d} x .
$$

3.5. Example of a random 1-current in the strict sense and its expectation. Let $\left(X_{t}, V_{t}\right)$ be the solution of the stochastic equation in $\mathbb{R}^{d} \times \mathbb{R}^{d}$ :

$$
\begin{aligned}
\mathrm{d} X_{t} & =V_{t} \mathrm{~d} t, \\
\mathrm{~d} V_{t} & =b\left(t, X_{t}, V_{t}\right) \mathrm{d} t+\mathrm{d} W_{t},
\end{aligned}
$$


where $b$ is Lipschitz continuous and $W_{t}$ is a Brownian motion in $\mathbb{R}^{d}$. Assume that $\left(X_{0}, V_{0}\right)$ has a smooth density $p_{0}(x, v)$ with respect to Lebesgue measure. Thanks to hypoellipticity, $\left(X_{t}, V_{t}\right)$ has, for every $t>0$, a smooth density $p_{t}(x, v)$ with respect to Lebesgue measure; it is a solution to the Fokker-Planck equation:

$$
\begin{aligned}
\frac{\partial p}{\partial t} & =\Delta_{v} p-v \cdot \nabla_{x} p-\operatorname{div}_{v}(b p), \\
\left.p\right|_{t=0} & =p_{0} .
\end{aligned}
$$

Given $T>0$, consider the random curve in $\mathbb{R}^{d}$ given by $t \in[0, T] \mapsto X_{t} \in \mathbb{R}^{d}$ (not necessarily simple) and consider the associated random 1-current $\xi_{X}$ formally defined as

$$
\xi_{X}(x)=\int_{0}^{T} \delta_{X_{t}}(x) V_{t} \mathrm{~d} t, \quad x \in \mathbb{R}^{d}
$$

namely

$$
\left(\xi_{X}, \theta\right)=\int_{0}^{T}\left\langle\theta\left(X_{t}\right), V_{t}\right\rangle \mathrm{d} t, \quad \theta \in C_{c}\left(\mathbb{R}^{d}, \mathbb{R}^{d}\right) .
$$

One can easily recognize that $\xi_{X}$ is a 1-current in the strict sense. We have

$$
\begin{aligned}
\mathbb{E}\left[\left(\xi_{X}, \theta\right)\right]=\mathbb{E}\left[\int_{0}^{T}\left\langle\theta\left(X_{t}\right), V_{t}\right\rangle \mathrm{d} t\right] & =\int_{0}^{T} \mathbb{E}\left[\left\langle\theta\left(X_{t}\right), V_{t}\right\rangle\right] \mathrm{d} t \\
& =\int_{0}^{T}\left(\int_{\mathbb{R}^{d} \times \mathbb{R}^{d}}\langle\theta(x), v\rangle p_{t}(x, v) \mathrm{d} x \mathrm{~d} v\right) \mathrm{d} t .
\end{aligned}
$$

We may also proceed as in Proposition 20, and see directly from (3-17) that, for any $x \in \mathbb{R}^{d}$, we do have formally

$$
\begin{aligned}
\mathbb{E}\left[\xi_{X}(x)\right] & =\int_{0}^{T} \mathbb{E}\left[\delta_{X_{t}}(x) V_{t}\right] \mathrm{d} t \\
& =\int_{0}^{T} \mathrm{~d} t \int_{\mathbb{R}^{d} \times \mathbb{R}^{d}} \delta_{y}(x) v p_{t}(y, v) \mathrm{d} y \mathrm{~d} v \\
& =\int_{0}^{T} \mathrm{~d} t \int_{\mathbb{R}^{d} \times \mathbb{R}^{d}} \delta_{x}(y) v p_{t}(y, v) \mathrm{d} y \mathrm{~d} v \\
& =\int_{0}^{T} \mathrm{~d} t \int_{\mathbb{R}^{d}} v p_{t}(x, v) \mathrm{d} v .
\end{aligned}
$$

In accordance with the above, we have proven the following.

Proposition 32. The 1-current $\xi_{X}$ admits a mean current $E\left[\xi_{X}\right]$ on $\mathbb{R}^{d}$, induced by the vector field

$$
u(x)=\int_{0}^{T} \int_{\mathbb{R}^{d}} v p_{t}(x, v) \mathrm{d} v \mathrm{~d} t, \quad x \in \mathbb{R}^{d}
$$


3.6. Example of a random 1-current in the broad sense and its expectation. Let $X_{t}$ be the solution of the stochastic equation in $\mathbb{R}^{d}$ :

$$
\mathrm{d} X_{t}=b\left(t, X_{t}\right) \mathrm{d} t+\mathrm{d} W_{t},
$$

where $b$ is Lipschitz continuous and $W_{t}$ is a Brownian motion in $\mathbb{R}^{d}$. Assume that $X_{0}$ has a smooth density $p_{0}(x)$ with respect to Lebesgue measure. Also $X_{t}$ has, for every $t>0$, a smooth density $p_{t}(x)$ with respect to Lebesgue measure; it is a solution to the Fokker-Planck equation:

$$
\begin{aligned}
\frac{\partial p}{\partial t} & =\Delta_{x} p-\operatorname{div}_{x}(b p), \\
\left.p\right|_{t=0} & =p_{0} .
\end{aligned}
$$

Given $T>0$, consider again the random curve $X_{t}$ in $\mathbb{R}^{d}$ (not necessarily simple) and consider the associated random 1-current $\xi_{X}$ formally defined as

$$
\xi_{X}(x)=\int_{0}^{T} \delta_{X_{t}}(x) \mathrm{d} X_{t}, \quad x \in \mathbb{R}^{d} ;
$$

namely,

$$
\left(\xi_{X}, \theta\right)=\int_{0}^{T}\left\langle\theta\left(X_{t}\right), \mathrm{d} X_{t}\right\rangle, \quad \theta \in C_{c}\left(\mathbb{R}^{d}, \mathbb{R}^{d}\right),
$$

where now the integral is understood as a stochastic integral. There are two main choices: Itô and Stratonovich integrals. Let us discuss only the Itô case, but the other one is also not difficult.

Given $\theta \in C_{c}\left(\mathbb{R}^{d}, \mathbb{R}^{d}\right),(\xi, \theta)$ is a well defined random variable, because the process $\theta\left(X_{t}\right)$ is adapted, $E\left[\int_{0}^{T}\left\|\theta\left(X_{t}\right)\right\|^{2} \mathrm{~d} t\right]<\infty$, and $\int_{0}^{T}\left\langle\theta\left(X_{t}\right), \mathrm{d} X_{t}\right\rangle$ is given by

$$
\int_{0}^{T}\left\langle\theta\left(X_{t}\right), \mathrm{d} X_{t}\right\rangle=\int_{0}^{T}\left\langle\theta\left(X_{t}\right), b\left(t, X_{t}\right)\right\rangle \mathrm{d} t+\int_{0}^{T}\left\langle\theta\left(X_{t}\right), \mathrm{d} W_{t}\right\rangle .
$$

So $\xi_{X}$ is a current in the broad sense. In general, it is not clear if it is a current also in the strict sense because we cannot "fix $\omega$ " and consider the map $\omega \in \Omega \mapsto$ $\int_{0}^{T}\left\langle\theta\left(X_{t}(\omega)\right), \mathrm{d} X_{t}(\omega)\right\rangle$ (the Itô integral is an equivalence class and its pointwise evaluation at $\omega$ is not a well-defined concept).

We have

$$
\mathbb{E}\left[\int_{0}^{T}\left\langle\theta\left(X_{t}\right), \mathrm{d} X_{t}\right\rangle\right]=\int_{0}^{T} \mathbb{E}\left[\left\langle\theta\left(X_{t}\right), b\left(t, X_{t}\right)\right\rangle\right] \mathrm{d} t+\mathbb{E}\left[\int_{0}^{T}\left\langle\theta\left(X_{t}\right), \mathrm{d} W_{t}\right\rangle\right] .
$$

Since for an Itô integral $E\left[\int_{0}^{T}\left\langle\theta\left(X_{t}\right), \mathrm{d} W_{t}\right\rangle\right]=0$, we have

$$
\int_{0}^{T} \mathbb{E}\left[\left\langle\theta\left(X_{t}\right), b\left(t, X_{t}\right)\right\rangle\right] \mathrm{d} t=\int_{0}^{T}\left(\int_{\mathbb{R}^{d}}\langle\theta(x), b(t, x)\rangle p_{t}(x) \mathrm{d} x\right) \mathrm{d} t .
$$

Hence we have proved the following. 
Proposition 33. The 1 -current $\xi_{X}$ admits a mean current $\mathbb{E}\left[\xi_{X}\right]$ on $\mathbb{R}^{d}$, induced by the vector field

$$
u(x)=\int_{0}^{T} b(t, x) p_{t}(x) \mathrm{d} t
$$

\section{Advanced applications and open problems}

4.1. A mathematical model for tumor induced angiogenesis. The main features of the process of formation of a tumor-driven vessel network are (see [Chaplain and Stuart 1993; Plank and Sleeman 2004; Bonilla et al. 2017])

(i) vessel branching;

(ii) vessel extension;

(iii) chemotaxis in response to a generic tumor angiogenic factor (TAF), released by tumor cells;

(iv) haptotactic migration in response to fibronectin gradient, emerging from the extracellular matrix and through degradation and production by endothelial cells themselves; and

(v) anastomosis, the coalescence of a capillary tip with an existing vessel.

We will limit ourselves to describe the dynamics of tip cells at the front of growing vessels, as a consequence of chemotaxis in response to a generic TAF released by tumor cells.

The $i$-th tip cell is characterized by its position and velocity $\left(X_{t}^{i}, V_{t}^{i}\right) \in \mathbb{R}^{2 d}$ for $t \geq 0$; also its history

$$
\left(X_{s}^{i}, V_{s}^{i}\right)_{s \in\left[T^{i}, \Theta^{i} \wedge t\right)}
$$

plays a role; the random variables $T^{i}$ and $\Theta^{i}$ are respectively the birth (branching) and death (anastomosis) times of the $i$-th tip cell. All random variables and processes are defined on a filtered probability space $\left(\Omega, \mathcal{F}, \mathcal{F}_{t}, P\right)$.

The number of tip cells changes in time, due to proliferation and death. We denote this random number by $N_{t}$ for $t \geq 0$.

The growth factor is a random function $C: \Omega \times[0, \infty) \times \mathbb{R}^{d} \rightarrow \mathbb{R}$, that we write as $C(t, x)$.

Tip cells and growth factor satisfy the system

$$
\begin{aligned}
\mathrm{d} X_{t}^{i} & =V_{t}^{i}, \\
\mathrm{~d} V_{t}^{i} & =\left[-k_{1} V_{t}^{i}+f\left(C\left(t, X_{t}^{i}\right)\right) \nabla C\left(t, X_{t}^{i}\right)\right] \mathrm{d} t+\sigma \mathrm{d} W_{t}^{i}, \\
\frac{\partial}{\partial t} C(t, x) & =k_{2} \delta_{A}(x)+d_{1} \Delta C(t, x)-\eta(t, x, S .) C(t, x),
\end{aligned}
$$


where $k_{1}, k_{2}, \sigma, d_{1}>0$ and $f: \mathbb{R} \rightarrow \mathbb{R}$ are given, $W_{t}^{i}$ for $i \in \mathbb{N}$ are independent Brownian motions, $A$ is a Borel set of $\mathbb{R}^{d}$ representing the tumoral region acting as a source of the TAF; initial conditions $X_{0}^{i}, V_{0}^{i}$ and $C(0, x)$ are also given.

Let us describe the term $\eta(t, x, S$.). For every $t \geq 0$, we introduce the measure

$$
S_{t}:=\sum_{i=1}^{N_{t}} 1_{t \in\left[T^{i}, \Theta^{i}\right)} \epsilon_{\left(X_{t}^{i}, V_{t}^{i}\right)},
$$

where $\epsilon$ denotes the usual Dirac measure. With these notations we may assume that, for every $t \geq 0$, the function $\eta(t, \cdot, \cdot)$ maps $\mathbb{R}^{d} \times C\left([0, t] ; \mathcal{M}\left(\mathbb{R}^{d}\right)\right)$ into $\mathbb{R}$ :

$$
\eta(t, \cdot, \cdot): \mathbb{R}^{d} \times C\left([0, t] ; \mathcal{M}\left(\mathbb{R}^{d}\right)\right) \rightarrow \mathbb{R}
$$

With the notation $\eta\left(t, x, S\right.$.), we understand $\eta\left(t, x,\left\{S_{s}\right\}_{s \in[0, t]}\right)$.

We may leave the function $\eta$ unspecified, with suitable assumptions. However, to help with intuition, we may assume the following structure:

$$
\eta(t, x, S .)=\int_{0}^{t} e^{-(t-s) / \tau}\left(\int_{\mathbb{R}^{d}} \int_{\mathbb{R}^{d}} K\left(x-x^{\prime}\right)\left|v^{\prime}\right| S_{S}\left(\mathrm{~d} x^{\prime}, \mathrm{d} v^{\prime}\right)\right) \mathrm{d} s
$$

for a suitable function $K: \mathbb{R}^{d} \rightarrow \mathbb{R}$.

In the SDE system (4-1), besides the friction force, there is a force due to the underlying TAF field $C(t, \mathbf{x})$; from the relevant literature we take [Plank and Sleeman 2004; Stéphanou et al. 2006]

$$
f(C)=\frac{d_{1}}{\left(1+\gamma_{1} C\right)^{q}} .
$$

The capillary network of endothelial cells $X(t)$ consists of the union of all random trajectories representing the extension of individual capillary tips from the random time of birth (branching) $T^{i}$ to the random time of death (anastomosis) $\Theta^{i}$,

$$
X(t)=\bigcup_{i=1}^{N_{t}}\left\{X^{i}(s), T^{i} \leq s \leq \min \left\{t, \Theta^{i}\right\}\right\}
$$

giving rise to a stochastic network. Thanks to the choice of a Langevin model for the vessel's extension, we may assume that the trajectories are sufficiently regular and have integer Hausdorff dimension 1.

Hence the random measure [Capasso and Villa 2008]

$$
A \in \mathcal{B}_{\mathbb{R}^{d}} \mapsto \mathcal{H}^{1}(X(t) \cap A) \in \mathbb{R}_{+}
$$


may admit a random generalized density $\delta_{X(t)}(x)$ with respect to the usual Lebesgue measure on $\mathbb{R}^{d}$ such that, for any $A \in \mathcal{B}_{\mathbb{R}^{d}}$,

$$
\mathcal{H}^{1}(X(t) \cap A)=\int_{A} \delta_{X(t)}(x) \mathrm{d} x .
$$

By Theorem 11, we may then state that

$$
\mathcal{H}^{1}(X(t) \cap A)=\int_{0}^{t} \sum_{i=1}^{N_{s}} \epsilon_{X^{i}(s)}(A)\left|\frac{\mathrm{d}}{\mathrm{d} s} X^{i}(s)\right| \rrbracket_{s \in\left[T^{i}, \Theta^{i}\right)} \mathrm{d} s .
$$

Hence,

$$
\delta_{X(t)}=\int_{0}^{t} \sum_{i=1}^{N_{s}} \delta_{X^{i}(s)}\left|\frac{\mathrm{d}}{\mathrm{d} s} X^{i}(s)\right| \mathbb{q}_{s \in\left[T^{i}, \Theta^{i}\right)} \mathrm{d} s .
$$

4.1.1. Vessel branching. Two kinds of branching have been identified; either from a tip or from a vessel.

The birth process of new tips can be described in terms of a marked point process (see, e.g., [Brémaud 1981]), by means of the random measure $\Phi$ on $\mathcal{B}_{\mathbb{R}^{+} \times \mathbb{R}^{d} \times \mathbb{R}^{d}}$ such that, for any $t \geq 0$ and any $B \in \mathcal{B}_{\mathbb{R}^{d}} \times \mathbb{R}^{d}$,

$$
\Phi((0, t] \times B):=\int_{0}^{t} \int_{B} \Phi(\mathrm{d} s \times \mathrm{d} x \times \mathrm{d} v),
$$

where $\Phi(\mathrm{d} s \times \mathrm{d} x \times \mathrm{d} v)$ is the random variable that counts those tips born either from an existing tip, or from an existing vessel, during times in $(s, s+\mathrm{d} s]$, with positions in $(x, x+\mathrm{d} x]$, and velocities in $(v, v+\mathrm{d} v]$.

Given the history $\mathcal{F}_{t^{-}}$of the whole process up to time $t^{-}$, we claim that the compensator of the random measure $\Phi(\mathrm{d} s \times \mathrm{d} x \times \mathrm{d} v)$ is $\alpha(C(s, x)) G_{v_{0}}(v) S_{s}(d(x, v)) \mathrm{d} s$

$$
+\beta(C(s, x)) G_{v_{0}}(v)\left(K_{1} * \delta_{X(t)}\right)(x) \mathrm{d} x \mathrm{~d} v \mathrm{~d} s,
$$

where $\alpha(C)$ and $\beta(C)$ are nonnegative functions; for example, we may take

$$
\alpha(C)=\beta(C)=\alpha_{1} \frac{C}{C_{R}+C},
$$

where $C_{R}$ is a reference density parameter [Capasso and Morale 2009]; $K_{1}: \mathbb{R}^{d} \rightarrow$ $\mathbb{R}$ is a suitable mollifying kernel.

As a technical simplification, we will further assume that the initial value of the state of a new tip is $\left(T^{N(t)+1}, X^{N(t)+1}, v^{N(t)+1}\right)$, where $T^{N(t)+1}$ is the random time of branching, $X^{N(t)+1}$ is the random point of branching, and $v^{N(t)+1}$ is a random velocity, selected out of a normal distribution $G_{v_{0}}$ with mean $v_{0}$, and some variance. 
4.1.2. Anastomosis. When a vessel tip meets an existing vessel, it joins at that point and time and it stops moving. This process is called tip-vessel anastomosis.

As in the case of the branching process, we may model this process via a marked counting process; anastomosis is modeled as a "death" process.

Let $\Psi$ denote the random measure on $\mathcal{B}_{\mathbb{R}^{+} \times \mathbb{R}^{d} \times \mathbb{R}^{d}}$ such that, for any $t \geq 0$ and any $B \in \mathcal{B}_{\mathbb{R}^{d} \times \mathbb{R}^{d}}$,

$$
\Psi((0, t] \times B):=\int_{0}^{t} \int_{B} \Psi(\mathrm{d} s \times \mathrm{d} x \times \mathrm{d} v),
$$

where $\Psi(\mathrm{d} s \times \mathrm{d} x \times \mathrm{d} v)$ is the random variable counting those tips that are absorbed by the existing vessel network during time $(s, s+\mathrm{d} s]$, with position in $(x, x+\mathrm{d} x]$, and velocity in $(v, v+\mathrm{d} v]$.

We assume that the compensator of the random measure $\Psi(\mathrm{d} s \times \mathrm{d} x \times \mathrm{d} v)$ is

$$
\gamma\left(K_{1} * \delta_{X(s)}\right)(x) S_{S}(d(x, v)) \mathrm{d} s,
$$

where $\gamma$ is a suitable constant.

4.2. Turbulence. The topic of turbulence is too wide and deep to be recalled here; let us mention a general reference [Frisch 1995] and one example to attempt to develop fragments of a rigorous theory based on the stochastic Navier-Stokes equations and their invariant measures [Flandoli et al. 2008]. Among the several ideas to approach turbulence, which is to a wide extent a statistical theory, there is one of Alexander Chorin [1994] which is particularly attractive. Based on some evidence that turbulent fluids are "made" (this sentence has to be taken in a very intuitive sense) of vortex structures, in particular filament-like structures (see [Frisch 1995] for a discussion), Chorin had the idea to describe such "vortex filaments" by means of paths of stochastic processes, and relate the statistical properties of these processes to the statistical properties of turbulent fluids. In particular, Chorin considered the so called self-avoiding walk and tried to connect its Flory exponents to the Kolmogorov exponents of K41 theory of turbulence - we cannot enter in further detail here, see [Chorin 1994]. Unfortunately, as Chorin admitted in his book, this connection is not clearly identified, so it was only a research suggestion; taken by some authors, like Gallavotti [2002], Lions and Majda [2000] and Flandoli and Gubinelli [2002] who tried to develop part of such arguments using Brownian motion instead of self-avoiding walk. The problem remains essentially open after these contributions.

The concept of a random 1-current described in the present work is strongly related to - in fact, it was strongly motivated by — stochastic vortex filaments. It does not solve in itself the question of connection with K41 or other statistical theories of turbulence, but it gives a precise language to approach it. Before we 
continue, let us say again that vortex filaments are an intuitive concept, since classical continuum mechanics allows us only to define the vorticity field of a fluid, and the identification of "structures" in it is not rigorous, at least at present. Having said this, the intuitive idea of a vortex filament is a curve, a vortex line (an integral curve of the vorticity field) over which the vorticity field is supported - maybe with the generalization of a finite number of such lines, not only one. This directly leads to the concept of a 1-current, a distributional vector field, concentrated along curves. Since a statistical theory should be developed, random 1-currents are the right objects. If we denote by $X$ the process whose paths represent the vortex filaments, the random distributional vorticity field is given by

$$
\xi_{X}=\int_{0}^{T} \delta_{X_{s}} \mathrm{~d} X_{s}
$$

The two main open questions are about statistical properties and about the realism of these random vortex structures. Concerning the realism, we mean the connection between these structures and more classical objects of fluid dynamics, typically the partial differential equations of continuum mechanics. The only partial result until now in this direction is the mean field result [Bessaih et al. 2017], where a smoothed version of 3D Euler equations is obtained as the mean field of interacting vortex filaments - also with a smoothed Biot-Savart kernel.

Concerning the statistical properties, first attempts have been made in [Chorin 1994; Lions and Majda 2000; Flandoli and Gubinelli 2002] by using Gibbs measures. The problem, not yet solved, is to identify a stochastic process $X$ such that the random distributional vorticity field $\xi_{X}$ written above has statistical properties like those of K41 theory. This means that the associated random velocity field $u_{X}=K * \xi_{X}$ has to be introduced ( $K$ denotes here the Biot-Savart kernel) and expected values like

$$
\mathbb{E}\left[|u(x+r e)-u(x)|^{2}\right],
$$

where $r$ is a real number and $e$ is a unitary vector, have to be computed and compared with the prescription of statistical theories of turbulence (K41 prescribes a behavior of the form $\mathrm{Cr}^{2 / 3}$ for small $r$ ).

A more modest but still quite open problem, very related to the machinery developed in the present work, is the following one: given a vorticity field $\xi(x)-$ generic, or typical of turbulent fluids - find a stochastic process $X$ such that

$$
\xi(x)=\mathbb{E}\left[\xi_{X}\right](x) .
$$

Solving this problem may increase some understanding of the statistical problem above and provide relevant initial conditions for the mean-field approach of [Bessaih et al. 2017]. 
4.3. Pathwise stochastic analysis. In recent years, a new direction in stochastic analysis grew up thanks to a number of outstanding contributions. The beginning of this direction is often traced back to a paper by Hans Föllmer [1981] where he derived an Itô formula for the composition $f\left(x_{t}\right)$ of a $C^{2}$ function $f: \mathbb{R}^{d} \rightarrow \mathbb{R}$ with a single deterministic path $x:[0, T] \rightarrow \mathbb{R}^{d}$, which possesses quadratic variation along a sequence of partitions $\left(\pi_{n}\right)$ of $[0, T]$, in the sense that

$$
\left[x_{.}^{(i)}\right]_{t}^{\left(\pi_{n}\right)}:=\lim _{n \rightarrow \infty} \sum_{t_{i} \in \pi_{n}}\left(x_{t_{i+1} \wedge t}^{(i)}-x_{t_{i} \wedge t}^{(i)}\right)^{2}
$$

exists finite and continuous in $t \in[0, T]$, for each $i=1, \ldots, d$, where $x_{t}^{(i)}$ denotes the $i$-coordinate of $x_{t}$. The stochastic integral $\int_{0}^{t}\left\langle\nabla f\left(x_{s}\right), \mathrm{d} x_{s}\right\rangle$ required in the formula,

$$
f\left(x_{t}\right)-f\left(x_{0}\right)-\frac{1}{2} \int_{0}^{t} \sum_{i, j=1}^{d} \partial_{i} \partial_{j} f\left(x_{s}\right) \mathrm{d}\left[x_{\cdot}^{(i)}, x_{\cdot}^{(j)}\right]_{s}^{\left(\pi_{n}\right)}=\int_{0}^{t}\left\langle\nabla f\left(x_{s}\right), \mathrm{d} x_{s}\right\rangle,
$$

is not a priori defined, but it exists from the formula itself: the limit or Riemann sums

$$
\lim _{n \rightarrow \infty} \sum_{t_{i} \in \pi_{n}}\left\langle\nabla f\left(x_{t_{i} \wedge t}\right), x_{t_{i+1} \wedge t}-x_{t_{i} \wedge t}\right\rangle
$$

exists since the sum of the other terms in the formula have a limit, thanks to the existence of the quadratic variation (the so-called joint quadratic variation between two coordinates $\left[x^{(i)}, x^{(j)}\right]_{t}^{\left(\pi_{n}\right)}$ exists by polarization and it is a bounded variation function, hence the integral on the left-hand side of (4-11) exists as limit of RiemannStieltjes sums). Probability is not totally excluded by this approach, but its role is localized: probability provides the existence of the quadratic variation $\left[x^{(i)}\right]_{t}^{\left(\pi_{n}\right)}$ for almost every path of relevant stochastic processes, like Brownian motion and more generally continuous semimartingales; it is otherwise very difficult to construct a deterministic function having nonzero quadratic variation $\left[x^{(i)}\right]_{t}^{\left(\pi_{n}\right)}$ (if it is zero, the formula above is just the usual chain rule). In a sense, a main topic in stochastic analysis, which previously has been treated by probability from the first to the last step (namely Itô's formula from the viewpoint of Itô's approach), is now decoupled: one half of the story is based on probability - the existence of the quadratic variation - and the other half is purely deterministic.

The question arisen by that paper is: to what extent can one develop a similar approach for other pieces of stochastic analysis? A breakthrough is rough path calculus developed by Terry Lyons [1998], which introduces new classes of paths, defines stochastic integrals for them and solves stochastic differential equations. Again, the theory is fully deterministic but at the foundation there is the concept of rough path, a sort of path $x$ enriched by its Lévy areas $\int_{0}^{t} x_{s}^{(i)} \mathrm{d} x_{s}^{(j)}$ (we refer here to 
a subclass for the general theory); the latter object exists for almost every path of Brownian motion and semimartingales using probability, namely using Itô's theory of stochastic integration.

The theory of rough paths has been revisited with a novel approach by Massimiliano Gubinelli [2004] and, in a sense based on this new viewpoint, a sort of multidimensional analog (namely for functions $x: \mathbb{R}^{d} \rightarrow \mathbb{R}$ or more precisely for distributions) has been developed in the outstanding work of Martin Hairer [2014], see also the alternative theory by Gubinelli et al. [2015]. Again in these theories, probability guarantees the existence of fundamental objects, after which the procedure to define other objects and solve (ordinary or partial) differential equations is fully deterministic. Let us also mention this kind of two-step approach in other recent directions of stochastic analysis, like stochastic homogenization [Gloria and Otto 2015].

After reviewing this introduction to the subject of "stochastic calculus without probability", let us mention an open research direction related to the topics of the present paper. Probability allows us to define stochastic integrals of the form

$$
\int_{0}^{t}\left\langle\theta\left(X_{s}\right), \mathrm{d} X_{s}\right\rangle
$$

for several stochastic processes $X$ and functions $\theta$. The map $\theta \mapsto \int_{0}^{t}\left\langle\theta\left(X_{s}\right), \mathrm{d} X_{s}\right\rangle$ is a random 1-current in the broad sense. In many cases, thanks to probabilistic estimates, there is a random 1-current in the strict sense $\xi_{X, t}(\omega)$ associated to it, given by

$$
\xi_{X, t}=\int_{0}^{t} \delta_{X_{s}} \mathrm{~d} X_{s}
$$

This is, for a given $\omega$, a deterministic 1-current associated to the deterministic path $X .(\omega)$. Conceptually, this is similar to the quadratic variation or the Lévy area associated to $X .(\omega)$ : concepts which are well-defined by probability, and would be extremely difficult to define without. The question then is: starting from the deterministic pair

$$
\left(X .(\omega), \xi_{X, \cdot}(\omega)\right)
$$

is it possible to develop, by purely deterministic methods, some pieces of stochastic calculus, as it was done by the theories recalled above starting from the pairs (path, quadratic variation), (path, Lévy area) and so on? For instance, is it possible to formulate and solve differential equations driven by single paths of Brownian motions? At present, this program has not been developed. Only the regularity, in terms of distributions, of $\xi_{X, \cdot}(\omega)$ has been partially understood, see [Flandoli et al. 2005; 2009]. 


\section{Acknowledgements}

It is our duty and pleasure to acknowledge the generous contributions of Elisabetta Dejana (Universitá degli Studi di Milano, Italy) for Figures 5, 6, and Michael E. Kassner (University of Southern California, USA) for Figure 3. We are grateful to the anonymous referee for comments and suggestions that have led to a significant improvement of the paper.

\section{References}

[Ambrosio et al. 2000] L. Ambrosio, N. Fusco, and D. Pallara, Functions of bounded variation and free discontinuity problems, Clarendon Press, New York, 2000.

[Ambrosio et al. 2009] L. Ambrosio, V. Capasso, and E. Villa, "On the approximation of mean densities of random closed sets", Bernoulli 15:4 (2009), 1222-1242.

[Anderson 2003] A. R. A. Anderson, "The effects of cell adhesion on solid tumour geometry", pp. 315-325 in Morphogenesis and pattern formation in biological systems: experiments and models, edited by T. Sekimura et al., Springer, Tokyo, 2003.

[Araujo and Giné 1980] A. Araujo and E. Giné, The central limit theorem for real and Banach valued random variables, Wiley, New York, 1980.

[Baddeley and Molchanov 1997] A. J. Baddeley and I. S. Molchanov, "On the expected measure of a random set", pp. 3-20 in Proceedings of the international symposium on advances in theory and applications of random sets (Fontainebleau, France), edited by D. Jeulin, World Scientific, River Edge, NJ, 1997.

[Baddeley et al. 2007] A. Baddeley, I. Bárány, R. Schneider, and W. Weil, Stochastic geometry, Lecture Notes in Mathematics 1892, Springer, Heidelberg, 2007.

[Bessaih et al. 2017] H. Bessaih, M. Coghi, and F. Flandoli, "Mean field limit of interacting filaments and vector valued non linear PDEs", J. Stat. Phys. (published online January 2017).

[Bharucha-Reid 1972] A. T. Bharucha-Reid, Random integral equations, Mathematics in Science and Engineering 26, Academic Press, New York, 1972.

[Bonilla et al. 2017] L. L. Bonilla, V. Capasso, M. Alvaro, M. Carretero, and F. Terragni, "On the mathematical modelling of tumor-induced angiogenesis", Math. Biosci. Eng. 14:1 (2017), 45-66.

[Bosq 2000] D. Bosq, Linear processes in function spaces: theory and applications, Lecture Notes in Statistics 149, Springer, New York, 2000.

[Brémaud 1981] P. Brémaud, Point processes and queues: martingale dynamics, Springer, New York, 1981.

[Buck 1956] R. C. Buck, Advanced calculus, McGraw-Hill, New York, 1956.

[Burger et al. 2002] M. Burger, V. Capasso, and C. Salani, "Modelling multi-dimensional crystallization of polymers in interaction with heat transfer", Nonlinear Anal-Real 3:1 (2002), 139-160.

[Callister Jr. 2007] W. Callister Jr., Materials science and engineering: an introduction, 7th ed., Wiley, New York, 2007.

[Camerlenghi et al. 2014] F. Camerlenghi, V. Capasso, and E. Villa, "On the estimation of the mean density of random closed sets”, J. Multivariate Anal. 125 (2014), 65-88.

[Capasso 2003] V. Capasso (editor), Mathematical modelling for polymer processing: polymerization, crystallization, manufacturing, Mathematics in Industry 2, Springer, Berlin, 2003. 
[Capasso and Micheletti 2006] V. Capasso and A. Micheletti, "Stochastic geometry and related statistical problems in biomedicine", pp. 35-69 in Complex systems in biomedicine, edited by A. Quarteroni et al., Springer, Milan, 2006.

[Capasso and Morale 2009] V. Capasso and D. Morale, "Stochastic modelling of tumour-induced angiogenesis", J. Math. Biol. 58:1-2 (2009), 219-233.

[Capasso and Villa 2006] V. Capasso and E. Villa, "On the continuity and absolute continuity of random closed sets", Stoch. Anal. Appl. 24:2 (2006), 381-397.

[Capasso and Villa 2007] V. Capasso and E. Villa, "On mean densities of inhomogeneous geometric processes arising in material science and medicine”, Image Anal. Stereol. 26:1 (2007), 23-36.

[Capasso and Villa 2008] V. Capasso and E. Villa, "On the geometric densities of random closed sets", Stoch. Anal. Appl. 26:4 (2008), 784-808.

[Capasso et al. 2013] V. Capasso, M. Gromov, A. Harel-Bellan, N. Morozova, and L. L. Pritchard (editors), Pattern formation in morphogenesis: problems and mathematical issues, Springer Proceedings in Mathematics 15, Springer, Heidelberg, 2013.

[Carmeliet and Jain 2000] P. Carmeliet and R. K. Jain, "Angiogenesis in cancer and other diseases", Nature 407:6801 (2000), 249-257.

[Chaplain and Stuart 1993] M. A. J. Chaplain and A. M. Stuart, "A model mechanism for the chemotactic response of endothelial cells to tumour angiogenesis factor", Math. Med. Bio. 10:3 (1993), $149-168$.

[Chorin 1994] A. J. Chorin, Vorticity and turbulence, Applied Mathematical Sciences 103, Springer, New York, 1994.

[Evans and Gariepy 1992] L. C. Evans and R. F. Gariepy, Measure theory and fine properties of functions, CRC Press, Boca Raton, FL, 1992.

[Falconer 1986] K. J. Falconer, The geometry of fractal sets, Cambridge Tracts in Mathematics 85, Cambridge University Press, 1986.

[Federer 1996] H. Federer, Geometric measure theory, Springer, Berlin, 1996.

[Flandoli and Gubinelli 2002] F. Flandoli and M. Gubinelli, "The Gibbs ensemble of a vortex filament", Probab. Theory Related Fields 122:3 (2002), 317-340.

[Flandoli et al. 2005] F. Flandoli, M. Gubinelli, M. Giaquinta, and V. M. Tortorelli, "Stochastic currents", Stoch. Process. Appl. 115:9 (2005), 1583-1601.

[Flandoli et al. 2008] F. Flandoli, M. Gubinelli, M. Hairer, and M. Romito, "Rigorous remarks about scaling laws in turbulent fluids", Comm. Math. Phys. 278:1 (2008), 1-29.

[Flandoli et al. 2009] F. Flandoli, M. Gubinelli, and F. Russo, "On the regularity of stochastic currents, fractional Brownian motion and applications to a turbulence model", Ann. Inst. Henri Poincaré Probab. Stat. 45:2 (2009), 545-576.

[Folland 1999] G. B. Folland, Real analysis: modern techniques and their applications, 2nd ed., Wiley, New York, 1999.

[Föllmer 1981] H. Föllmer, "Calcul d'Ito sans probabilités", Séminaire de probabilités de Strasbourg 15 (1981), 143-150.

[Frisch 1995] U. Frisch, Turbulence, Cambridge University Press, 1995.

[Galbis and Maestre 2012] A. Galbis and M. Maestre, Vector analysis versus vector calculus, Springer, New York, 2012.

[Gallavotti 2002] G. Gallavotti, Foundations of fluid dynamics, Springer, Berlin, 2002.

[Giaquinta and Modica 2009] M. Giaquinta and G. Modica, Mathematical analysis: an introduction to functions of several variables, Birkhäuser, Boston, 2009. 
[Giaquinta et al. 1998] M. Giaquinta, G. Modica, and J. Souček, Cartesian currents in the calculus of variations, I: Cartesian currents, Mathematics and Related Areas 37, Springer, Berlin, 1998.

[Gloria and Otto 2015] A. Gloria and F. Otto, "The corrector in stochastic homogenization: optimal rates, stochastic integrability, and fluctuations", 2015. arXiv 1510.08290

[Gubinelli 2004] M. Gubinelli, "Controlling rough paths”, J. Funct. Anal. 216:1 (2004), 86-140.

[Gubinelli et al. 2015] M. Gubinelli, P. Imkeller, and N. Perkowski, "Paracontrolled distributions and singular PDEs", Forum Math. Pi 3 (2015), e6, 75.

[Hairer 2014] M. Hairer, “A theory of regularity structures”, Invent. Math. 198:2 (2014), 269-504.

[Hochrainer et al. 2007] T. Hochrainer, M. Zaiser, and P. Gumbsch, "A three-dimensional continuum theory of dislocation systems: kinematics and mean-field formulation", Philos. Mag. 87:8-9 (2007), $1261-1282$.

[Kassner et al. 2000] M. Kassner, M.-T. Pérez-Prado, K. Vecchio, and M. Wall, "Determination of internal stresses in cyclically deformed copper single crystals using convergent-beam electron diffraction and dislocation dipole separation measurements", Acta Mater. 48:17 (2000), 4247-4254.

[Kolmogorov 1956] A. N. Kolmogorov, Foundations of the theory of probability, Chelsea Publishing, New York, 1956.

[Kolmogorov and Fomin 1970] A. N. Kolmogorov and S. V. Fomin, Introductory real analysis, Prentice-Hall, Englewood Cliffs, NJ, 1970.

[Lions and Majda 2000] P.-L. Lions and A. Majda, "Equilibrium statistical theory for nearly parallel vortex filaments", Comm. Pure Appl. Math. 53:1 (2000), 76-142.

[Lyons 1998] T. J. Lyons, "Differential equations driven by rough signals", Rev. Mat. Iberoamericana 14:2 (1998), 215-310.

[Matheron 1965] G. Matheron, Les Variables Regionalisées et leur Estimation, Masson, Paris, 1965.

[Matheron 1975] G. Matheron, Random sets and integral geometry, Wiley, New York, 1975.

[Morgan 1998] F. Morgan, Geometric measure theory: a beginner's guide, Academic Press, San Diego, CA, 1998.

[Plank and Sleeman 2004] M. J. Plank and B. D. Sleeman, "Lattice and non-lattice models of tumour angiogenesis", Bull. Math. Biol. 66:6 (2004), 1785-1819.

[Stéphanou et al. 2006] A. Stéphanou, S. R. McDougall, A. R. A. Anderson, and M. A. J. Chaplain, "Mathematical modelling of the influence of blood rheological properties upon adaptative tumourinduced angiogenesis", Math. Comput. Modelling 44:1-2 (2006), 96-123.

[Ubukata 2003] T. Ubukata, "Computer modeling of microscopic features of molluscan shells", pp. 355-367 in Morphogenesis and pattern formation in biological systems: experiments and models, edited by T. Sekimura et al., Springer, Tokyo, 2003.

[Vladimirov 1979] V. S. Vladimirov, Generalized functions in mathematical physics, Mir, Moscow, 1979.

[Zähle 1982] M. Zähle, "Random processes of Hausdorff rectifiable closed sets", Math. Nachr. 108 (1982), 49-72.

Received 7 Jun 2016. Revised 4 Sep 2016. Accepted 24 Oct 2016.

VINCENZO CAPASSO: vincenzo. capasso@unimi.it

ADAMSS and Department of Mathematics, University of Milan, Via Saldini 50, I-20133 Milano, Italy

FRANCO FLANDOLI: flandoli@dma.unipi.it

Department of Mathematics, University of Pisa, Largo Pontecorvo 5, I-56127 Pisa, Italy 
EDITORIAL BOARD

ANTONIO CARCATERRA

ERIC A. CARLEN

FRANCESCO DELL'ISOLA

RAFFAELE ESPOSITO

ALBERT FANNJIANG

Gilles A. FranCFORT

Pierangelo MARCATI

JEAN-JACQUES MARIGO

PETER A. MARKOWICH

MARTIN OSTOJA-STARZEWSKI

PIERRE SEPPECHER

DAVID J. STEIGMANN

PAUl STEINMANN

PierRe M. SuQueT

MANAGING EDITORS

MICOL AMAR

CORRADO LATTANZIO

ANGELA MADEO

MARTIN OSTOJA-STARZEWSKI

ADVISORY BOARD

ADNAN AKAY

Holm AltenBaCH

MICOL AMAR

HARM ASKES

TEODOR ATANACKOVIĆ

VICTOR BERDICHEVSKY

GUY BOUCHITTÉ

ANDREA BRAIDES

ROBERTO CAMASSA

MAURO CARFORE

ERIC DARVE

FELIX DARVE

ANNA DE MASI

GianPiEtro DEL Piero

EMMANUELE Di BENEDETTO

BERNOLD FIEDLER

IRENE M. GAMBA

DAVID Y. GAO

SERGEY GAVRILYUK

TIMOTHY J. HEALEY

DOMINIQUE JEULIN

ROGER E. KHAYAT

CORRADO LATTANZIO

ROBERT P. LIPTON

ANGELO LUONGO

ANGELA MADEO

JUAN J. MANFREDI

CARLO MARCHIORO

GÉRARD A. MAUGIN

ROBERTO NATALINI PATRIZIO NEFF

ANDREY PIATNITSKI

ERRICO PRESUTTI

MARIO PULVIRENTI

LUCIO RUSSO

Miguel A. F. SANJUAN

PATRICK SElVADURAI

ALEXANDER P. SEYRANIAN

MIROSLAV ŠILHAVÝ

GUIDO SWEERS

ANTOINETTE TORDESILLAS

LEV TRUSKINOVSKY

JUAN J. L. VELÁZQUEZ VINCENZO VESPRI ANGELO VULPIANI msp.org/memocs

Università di Roma "La Sapienza", Italia

Rutgers University, USA

(CO-CHAIR) Università di Roma "La Sapienza", Italia

(TREASURER) Università dell'Aquila, Italia

University of California at Davis, USA

(CO-CHAIR) Université Paris-Nord, France

Università dell'Aquila, Italy

École Polytechnique, France

DAMTP Cambridge, UK, and University of Vienna, Austria

(CHAIR MANAGING EDITOR) Univ. of Illinois at Urbana-Champaign, USA

Université du Sud Toulon-Var, France

University of California at Berkeley, USA

Universität Erlangen-Nürnberg, Germany

LMA CNRS Marseille, France

Università di Roma "La Sapienza", Italia

Università dell'Aquila, Italy

Université de Lyon-INSA (Institut National des Sciences Appliquées), France

(CHAIR MANAGING EDITOR) Univ. of Illinois at Urbana-Champaign, USA

Carnegie Mellon University, USA, and Bilkent University, Turkey

Otto-von-Guericke-Universität Magdeburg, Germany

Università di Roma "La Sapienza", Italia

University of Sheffield, UK

University of Novi Sad, Serbia

Wayne State University, USA

Université du Sud Toulon-Var, France

Università di Roma Tor Vergata, Italia

University of North Carolina at Chapel Hill, USA

Università di Pavia, Italia

Stanford University, USA

Institut Polytechnique de Grenoble, France

Università dell'Aquila, Italia

Università di Ferrara and International Research Center MEMOCS, Italia

Vanderbilt University, USA

Freie Universität Berlin, Germany

University of Texas at Austin, USA

Federation University and Australian National University, Australia

Université Aix-Marseille, France

Cornell University, USA

École des Mines, France

University of Western Ontario, Canada

Università dell' Aquila, Italy

Louisiana State University, USA

Università dell'Aquila, Italia

Université de Lyon-INSA (Institut National des Sciences Appliquées), France University of Pittsburgh, USA

Università di Roma "La Sapienza”, Italia

Université Paris VI, France

Istituto per le Applicazioni del Calcolo "M. Picone", Italy

Universität Duisburg-Essen, Germany

Narvik University College, Norway, Russia

Università di Roma Tor Vergata, Italy

Università di Roma "La Sapienza”, Italia

Università di Roma “Tor Vergata”, Italia

Universidad Rey Juan Carlos, Madrid, Spain

McGill University, Canada

Moscow State Lomonosov University, Russia

Academy of Sciences of the Czech Republic

Universität zu Köln, Germany

University of Melbourne, Australia

École Polytechnique, France

Bonn University, Germany

Università di Firenze, Italia

Università di Roma La Sapienza, Italia

MEMOCS (ISSN 2325-3444 electronic, 2326-7186 printed) is a journal of the International Research Center for the Mathematics and Mechanics of Complex Systems at the Università dell'Aquila, Italy.

Cover image: "Tangle” by $\odot$ John Horigan; produced using the Context Free program (contextfreeart.org).

PUBLISHED BY

7 mathematical sciences publishers

nonprofit scientific publishing

http://msp.org/

(C) 2016 Mathematical Sciences Publishers 
Special issue in honor of

Lucio Russo

Lucio Russo: A multifaceted life

Raffaele Esposito and Francesco dell'Isola

The work of Lucio Russo on percolation Geoffrey R. Grimmett

"Mathematics" and "physics" in the science of harmonics Stefano Isola

From quantum to classical world: emergence of trajectories in a quantum system Rodolfo Figari and Alessandro Teta

Propagation of chaos and effective equations in kinetic theory: a brief survey Mario Pulvirenti and Sergio Simonella

What decides the direction of a current? Christian Maes

A remark on eigenvalue perturbation theory at vanishing isolation distance Fiorella Barone and Sandro Graffi

Some results on the asymptotic behavior of finite connection probabilities in percolation Massimo Campanino and Michele Gianfelice

Correlation inequalities for the Potts model Geoffrey R. Grimmett

Quantum mechanics: some basic techniques for some basic models, I: The models Vincenzo Grecchi

Quantum mechanics: some basic techniques for some basic models, II: The techniques Vincenzo Grecchi

On stochastic distributions and currents

Vincenzo Capasso and Franco Flandoli

A note on Gibbs and Markov random fields with constraints and their moments Alberto Gandolfi and Pietro Lenarda

Quantum mechanics: light and shadows (ontological problems and epistemic solutions) Gianfausto Dell'Antonio

MEMOCS is a journal of the International Research Center for the Mathematics and Mechanics of Complex Systems at the Università dell' Aquila, Italy.

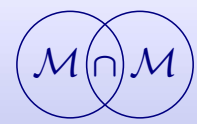

